\title{
Light-cone gauge and the calculation of the two-loop splitting functions
}

\author{
A. Bassetto, ${ }^{1}$ G. Heinrich, ${ }^{2}$ Z. Kunszt, ${ }^{2}$ and W. Vogelsang ${ }^{3}$ \\ ${ }^{1}$ Dipartimento di Fisica “G. Galilei,"” via Marzolo 8, I-35131 Padova, Italy \\ and INFN, Sezione di Padova, Padova, Italy \\ ${ }^{2}$ Institute of Theoretical Physics, ETH Zürich, Switzerland \\ ${ }^{3}$ Theoretical Physics Division, CERN, CH-1211 Geneva 23, Switzerland
}

(Received 12 May 1998; published 1 October 1998)

\begin{abstract}
We present calculations of next-to-leading order QCD splitting functions, employing the light-cone gauge method of Curci, Furmanski, and Petronzio (CFP). In contrast with the "principal-value" prescription used in the original CFP paper for dealing with the poles of the light-cone gauge gluon propagator, we adopt the Mandelstam-Leibbrandt prescription which is known to have a solid field-theoretical foundation. We find that indeed the calculation using this prescription is conceptually clear and avoids the somewhat dubious manipulations of the spurious poles required when the principal-value method is applied. We reproduce the wellknown results for the flavor nonsinglet splitting function and the $N_{C}^{2}$ part of the gluon-to-gluon singlet splitting function, which are the most complicated ones, and which provide an exhaustive test of the ML prescription. We also discuss in some detail the $x=1$ end point contributions to the splitting functions. [S0556-2821(98)03219-6]

PACS number(s): 12.38.Bx
\end{abstract}

\section{INTRODUCTION}

The advantages of working in axial gauges when performing perturbative QCD calculations have been known for a long time [1]. Those gauges enable us to retain, in higher order calculations, a natural "partonic" interpretation for the vector field, typical to a leading logarithmic approximation.

Among axial gauges, the one which enjoys a privileged status is the light-cone axial (LCA) gauge, characterized by the condition $n^{\mu} A_{\mu}=0, n^{\mu}$ being a lightlike vector $\left(n^{2}\right.$ $=0)$. At variance with temporal $\left(n^{2}>0\right)$ and spacelike $\left(n^{2}\right.$ $<0)$ axial gauges, which do have problems already at the free level [2], and with the spacelike "planar" gauge [1] in which the behavior of the theory to higher loop orders is still unsettled [2], the LCA gauge can be canonically quantized [3] and renormalized [4] at all orders in the loop expansion following a well-established procedure. To reach this goal it is crucial to treat the "spurious" singularity occurring in the tensorial part of the vector propagator,

$$
\mathcal{D}^{\mu \nu}(l)=\frac{i}{l^{2}+i \varepsilon}\left(-g^{\mu \nu}+\frac{n^{\mu} l^{\nu}+n^{\nu} l^{\mu}}{n l}\right),
$$

according to a prescription independently suggested by Mandelstam [5] and Leibbrandt [6] (ML) and derived in Ref. [3] in the context of equal-time canonical quantization:

$$
\frac{1}{(n l)} \rightarrow \frac{1}{[n l]} \equiv \frac{1}{n l+i \varepsilon \operatorname{sgn}\left(n^{*} l\right)}=\frac{n^{*} l}{n^{*} \ln l+i \varepsilon},
$$

the two expressions being equal in the sense of the theory of distributions. The vector $n^{*}$ is lightlike, and such that $n^{*} n$ $=1$. Denoting by $l_{\perp}$ the transverse part of the vector $l_{\mu}$, orthogonal to both $n_{\mu}$ and $n_{\mu}^{*}$, one has

$$
2(n l)(n * l)=l^{2}+l_{\perp}^{2} .
$$

The key feature of the ML prescription is that the spurious poles in the complex $l_{0}$ plane are placed in the second and fourth quadrants, i.e., with the same pattern as one encounters for the usual covariant denominators such as $1 /\left(l^{2}\right.$ $+i \varepsilon)$. One can therefore perform a proper Wick rotation to Euclidean momenta, and a suitable power counting criterion in the Euclidean integrals will give information on the ultraviolet (UV) divergencies of the corresponding Minkowskian integrals. This is in contrast to the Cauchy principal-value $(\mathrm{PV})$ prescription, which, under a Wick rotation, entails further contributions and therefrom a violation of power counting.

A crucial property of the ML distribution is the occurrence of two contributions with opposite signs in the absorptive part of the vector propagator [7]:

$$
\begin{aligned}
\operatorname{disc} & {\left[\mathcal{D}_{\mu \nu}(l)\right] } \\
= & 2 \pi \delta\left(l^{2}\right) \Theta\left(l_{0}\right)\left(-g_{\mu \nu}+\frac{2 n^{*} l}{n^{*} n} \frac{n_{\mu} l_{\nu}+n_{\nu} l_{\mu}}{l_{\perp}^{2}}\right) \\
& -2 \pi \delta\left(l^{2}+l_{\perp}^{2}\right) \Theta\left(l_{0}\right) \frac{2 n^{*} l}{n^{*} n} \frac{n_{\mu} l_{\nu}+n_{\nu} l_{\mu}}{l_{\perp}^{2}} .
\end{aligned}
$$

Here the second, ghostlike, contribution (which is not present in the PV prescription) is responsible for the milder infrared (IR) behavior of the ML propagator. The presence of this axial ghost was stressed in [8]; its properties are exhaustively discussed in [2]. Clearly, if one has a cut diagram with, say, $m$ final-state gluons, there is a discontinuity like Eq. (4) for each of the gluons: i.e., the phase space will split up into $2^{m}$ pieces.

One of the most interesting and nontrivial applications of the LCA gauge is the computation of the (spin-independent) splitting functions for the two-loop Altarelli-Parisi (AP) evolution of parton densities, following a method proposed and 
used by Curci, Furmanski, and Petronzio (CFP) in Refs. $[9,10]$. This method is based on the observation [11] that in axial gauges the two-particle-irreducible kernels of the ladder diagrams are finite, so that the collinear singularities that give rise to parton evolution only originate from the lines connecting the kernels. Therefrom, using renormalization group techniques, the splitting functions are obtained by some suitable projection of the ladder diagrams, exploiting the factorization theorem of mass singularities [11]. We refrain from giving further details of the CFP method, since these can be found in $[9,12]$. We just mention at this point that one projects on the quantity $\Gamma_{i j}$, given by

$$
\begin{aligned}
\Gamma_{i j}\left(x, \alpha_{s}, \frac{1}{\epsilon}\right)= & Z_{j}\left[\delta(1-x) \delta_{i j}+x \operatorname{PP} \int \frac{d^{d} k}{(2 \pi)^{d}}\right. \\
& \left.\times \delta\left(x-\frac{n k}{p n}\right) U_{i} K \frac{1}{1-\mathcal{P} K} L_{j}\right],
\end{aligned}
$$

where $2 \epsilon=(4-d)$ and $K$ is a two-particle-irreducible (2PI) kernel, which is finite in the light-cone gauge $[11,9]$. The labels $i, j$ run over quarks and gluons; in the flavor nonsinglet case one has just $\{i j\}=\{q q\}$. Furthermore, in Eq. (5), PP denotes the pole part, and the projectors $U_{i}, L_{i}$ are given by

$$
\begin{aligned}
& U_{q}=\frac{1}{4 n k} \not h, \quad L_{q}=p, \\
& U_{g}=-g^{\mu \nu}, \quad L_{g}=\frac{1}{d-2}\left[-g^{\mu \nu}+\frac{n^{\mu} p^{\nu}+n^{\nu} p^{\mu}}{p n}\right] .
\end{aligned}
$$

The splitting functions $P_{i j}$ to the desired order can be read off from the $1 / \epsilon$ pole of $\Gamma_{i j}$ :

$$
\begin{aligned}
\Gamma_{i j}\left(x, \alpha_{s}, \frac{1}{\epsilon}\right)= & \delta(1-x) \delta_{i j} \\
& -\frac{1}{\epsilon}\left(\frac{\alpha_{s}}{2 \pi} P_{i j}^{(0)}(x)+\frac{1}{2}\left(\frac{\alpha_{s}}{2 \pi}\right)^{2} P_{i j}^{(1)}(x)+\ldots\right) \\
& +O\left(\frac{1}{\epsilon^{2}}\right) .
\end{aligned}
$$

For future reference, we write down a similar expression [9] for the residue $Z_{j}(j=q, g)$ of the pole of the full quark (or gluon) propagator:

$$
\begin{aligned}
Z_{j}= & 1-\frac{1}{\epsilon}\left(\frac{\alpha_{s}}{2 \pi} \xi_{j}^{(0)}(x)+\frac{1}{2}\left(\frac{\alpha_{s}}{2 \pi}\right)^{2} \xi_{j}^{(1)}(x)+\ldots\right) \\
& +O\left(\frac{1}{\epsilon^{2}}\right) .
\end{aligned}
$$

Inspecting Eqs. (5),(7),(8), we see that $Z_{q}$ and $Z_{g}$ contribute to the end point $[\sim \delta(1-x)]$ parts of the splitting functions $P_{q q}$ and $P_{g g}$, respectively.
In the above references $[9,10]$, the "spurious" singularity $1 / n l$ of the gluon propagator was handled according to the PV prescription. The method of $[9,10]$ has been very successful in providing the first correct result for the next-toleading order (NLO) gluon-to-gluon splitting function. The result previously obtained in the operator product expansion (OPE) method [14] was not correct due to a subtle conceptual problem which was recently clarified $[15,16]$. The new Feynman gauge OPE calculations confirmed the old CFP result. Despite this success, the LCA calculation with the PV prescription is considered dubious because of the difficulties with power counting and the Wick rotation mentioned above. In particular it is not clear whether its "calculational rules" remain valid in higher orders. We note that the precise description of some of the new high precision collider data call for the extension of the NLO QCD analysis to next-to-nextto-leading order (NNLO). Therefore a deeper understanding of the formal field-theoretical basis of the CFP method is strongly motivated. The use of PV is by no means mandatory in the CFP method; it can also be applied when handling the $1 / l n$ singularities with the theoretically more sound ML prescription.

A first attempt using the ML prescription in connection with the CFP method has been performed in Ref. [7], where the one-loop AP splitting functions [13] have been correctly reproduced, both for the flavor nonsinglet and flavor singlet cases. A new characteristic feature of this calculation is that "real" and "virtual" contributions are separately well defined in the limit $x \rightarrow 1, x$ being the longitudinal momentum fraction, at variance with the corresponding PV result. This occurs thanks to the presence of the "axial" ghost, which, standing by the usual gluon term, protects its singular behavior with respect to the transverse momentum. There is no need of any IR cutoff to regularize intermediate results.

Beyond one loop, the calculation of the splitting functions, according to the CFP method in the LCA gauge with the ML prescription, has already been tackled in a recent paper [17]. We believe, however, that improvements to the calculation [17] can and should be made. First of all, only the $C_{F}^{2}$ part of the flavor nonsinglet splitting function is studied in [17]. In this paper we will also calculate the $C_{F} T_{f}$ part and, in particular, the far more complicated piece $\sim C_{F} N_{C}$ of this function, as well as the $N_{C}^{2}$ part of the gluon-to-gluon splitting function contributing to the flavor singlet sector. As we will show, this set of functions we consider comprises all possible one-loop structures of QCD and thus enables an exhaustive test of the ML prescription in this application. The ML calculation of the other singlet splitting functions, like the nondiagonal quark-to-gluon (and vice versa) one, is therefore not really required in this context: they will certainly come out correctly if the prescription works for the far more complicated cases we study.

Second, the power and virtues of the ML prescription were not fully exploited in [17], where some contributions resulting from the axial ghosts of the ML prescription were neglected. These contributions are $\sim \delta(1-x)$ and thus only affect the end points of the diagonal splitting functions. Nevertheless, their inclusion is required for a complete analysis, since only then can the crucial question of the finiteness of 
the 2PI kernels in the light-cone gauge be fully answered. We also remind the reader in this context that in the original CFP papers $[9,10]$, the end point contributions to the diagonal splitting functions were never determined by explicit calculation, but were derived in an indirect way from fermion number and energy-momentum conservation. The fact that we pay more attention to the point $x=1$ will enable us to improve this situation to a certain extent: for the first time within the CFP method, we will determine the full part $\sim C_{F} T_{f} \delta(1-x)$ of $P_{q q}^{V,(1)}$ by explicit calculation.

Finally, in [17] a principal-value regularization was still used at some intermediate steps of the calculation. Even though this was only done at places where it seemed a safe and well-defined procedure, it is more in the spirit of the ML prescription to abandon the PV completely and to stick to one single regularization, the dimensional one. This view is corroborated by the observation that the PV regularization as used in [17] actually turns out to become technically too complicated when one studies the $C_{F} N_{C}$ part of the flavor nonsinglet splitting function, or the $N_{C}^{2}$ part of $P_{g g}^{(1)}$.

The remainder of this paper is organized as follows: to set the framework, we will present a brief rederivation of the leading order (LO) quark-to-quark splitting function $P_{q q}^{(0)}$ in Sec. II. Section III will contain the calculation of the flavor nonsinglet splitting function at two loops. More specifically, we will discuss in detail the treatment of the various virtualcut and real-cut contributions in Secs. III A and III B, respectively, while Sec. III C presents the final results of the calculation. In Sec. III D, we discuss the end point contributions and provide a sample calculation of a two-loop contribution to the quark self-energy in the LCA gauge with the ML prescription. Section IV deals with the calculation of the $N_{C}^{2}$ part of $P_{g g}^{(1)}$. Finally, we summarize our work in Sec. V.

\section{RECALCULATION OF THE LO SPLITTING FUNCTION}

As a first example, we will rederive the LO result for the flavor nonsinglet splitting function, using the ML prescription. This is a rather trivial calculation that nevertheless displays the main improvements provided by the use of the ML prescription. Furthermore, the virtual graphs in the NLO calculation have the LO kinematics, and so this section also serves to prepare the NLO calculation. We noted before that the LO example has already been worked out in [7] where collinear poles were regularized by taking the initial quark off shell, $p^{2}<0$, rather than by using dimensional regularization. This is perfectly fine at the LO level, but beyond LO it seems a forbidding task to keep $p^{2} \neq 0$, and in fact the underlying method of $[9,10]$ that we are employing has been set up in such a way that it relies on the use of dimensional regularization, yielding final results that correspond to the modified minimal subtraction $(\overline{\mathrm{MS}})$ scheme. It therefore seems a useful exercise to sketch the calculation of $P_{q q}^{(0)}$ in the ML prescription if dimensional regularization is used.

The Feynman diagrams contributing to $\Gamma_{q q}$ at LO are shown in Fig. 1. For the gluon polarization tensor in diagram (a) we need to insert the two parts of the ML discontinuity in

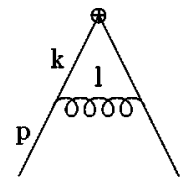

(a)

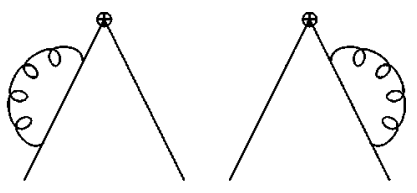

(b)
FIG. 1. Diagrams contributing to $\Gamma_{q q}$ at LO.

Eq. (4) with their two different $\delta$ functions. The first part of the phase space, resulting from $\delta\left(l^{2}\right)$, can be written as

$$
\begin{gathered}
x \int d^{d} k \int d^{d} l \delta\left(x-\frac{k n}{p n}\right) \delta(p-k-l) \delta\left(l^{2}\right) \\
=\frac{\pi^{1-\epsilon}}{2 \Gamma(1-\epsilon)} \int_{0}^{Q^{2}} d\left|k^{2}\right|\left(k_{\perp}^{2}\right)^{-\epsilon}
\end{gathered}
$$

where

$$
k_{\perp}^{2}=l_{\perp}^{2}=\left|k^{2}\right|(1-x) .
$$

The $\delta\left(l^{2}\right)$ contribution of graph (a) to $\Gamma_{q q}$ is then given by

$$
\begin{aligned}
\Gamma_{q q}^{(a), \delta\left(l^{2}\right)}(x)= & \frac{\alpha_{s}}{2 \pi} \mathrm{PP} \int_{0}^{Q^{2}} d\left|k^{2}\right|\left|k^{2}\right|^{-1-\epsilon} \\
& \times C_{F}(1-x)^{-\epsilon} \frac{1+x^{2}}{1-x} .
\end{aligned}
$$

Using the identity

$$
\begin{aligned}
(1-x)^{-1-\epsilon} \equiv & -\frac{1}{\epsilon} \delta(1-x)+\frac{1}{(1-x)_{+}} \\
& -\epsilon\left(\frac{\ln (1-x)}{1-x}\right)_{+}+\mathcal{O}\left(\epsilon^{2}\right),
\end{aligned}
$$

where the "plus" prescription is defined in the usual way, one readily obtains

$$
\begin{aligned}
\Gamma_{q q}^{(a), \delta\left(l^{2}\right)}(x)= & \frac{\alpha_{s}}{2 \pi} \operatorname{PP} \int_{0}^{Q^{2}} d\left|k^{2}\right|\left|k^{2}\right|^{-1-\epsilon} \\
& \times C_{F}\left[-\frac{2}{\epsilon} \delta(1-x)+\frac{1+x^{2}}{(1-x)_{+}}\right] .
\end{aligned}
$$

For the ghostlike part we introduce the variable $\kappa$ as

$$
k_{\perp}^{2}=l_{\perp}^{2}=-l^{2}=\left|k^{2}\right| \kappa .
$$

The phase space is then given by 


$$
\begin{aligned}
\int & d^{d} k \int d^{d} l \delta\left(x-\frac{k n}{p n}\right) \delta(p-k-l) \delta\left(l^{2}+l_{\perp}^{2}\right) \\
= & \frac{\pi^{1-\epsilon}}{2 \Gamma(1-\epsilon)} \int_{0}^{Q^{2}} d\left|k^{2}\right| \int_{0}^{1} d \kappa\left(k_{\perp}^{2}\right)^{-\epsilon} \\
& \times \delta((1-x)(1-\kappa)) \\
= & \frac{\pi^{1-\epsilon}}{2 \Gamma(1-\epsilon)} \delta(1-x) \int_{0}^{Q^{2}} d\left|k^{2}\right| \int_{0}^{1} \frac{d \kappa}{1-\kappa}\left(k_{\perp}^{2}\right)^{-\epsilon},
\end{aligned}
$$

where the last line follows since the root of the delta function for $\kappa=1$ never contributes when we insert the second term in Eq. (4) for the gluon polarization tensor into graph (a), thanks to the factor $2 n * l / l_{\perp}^{2} \sim(1-\kappa) / \kappa$ accompanying the $\delta\left(l^{2}+l_{\perp}^{2}\right)$ in Eq. (4). Thus, the ghost part contributes only at $x=1$. The contribution $\sim 1 / \kappa$ of $2 n^{*} l / l_{\perp}^{2}$ gives rise to a $1 / \epsilon$ pole in the final answer:

$$
\Gamma_{q q}^{(a), \delta\left(l^{2}+l_{\perp}^{2}\right)}(x)=\frac{\alpha_{s}}{2 \pi} \delta(1-x) \mathrm{PP} \int_{0}^{Q^{2}} d\left|k^{2}\right|\left|k^{2}\right|^{-1-\epsilon} C_{F}\left[\frac{2}{\epsilon}\right] .
$$

Adding Eqs. (13) and (16), we get the full contribution of graph (a) to $\Gamma_{q q}$ :

$$
\Gamma_{q q}^{(a)}(x)=\frac{\alpha_{s}}{2 \pi} \operatorname{PP} \int_{0}^{Q^{2}} d\left|k^{2}\right|\left|k^{2}\right|^{-1-\epsilon} C_{F} \frac{1+x^{2}}{(1-x)_{+}} .
$$

An important feature of this result should be emphasized, as it will also be encountered at NLO: the integrand in Eq. (17) is completely finite, in a distributional sense. In other words, using the ML prescription, we have verified the finiteness of the LO 2PI kernel $q \rightarrow q g$ in the light-cone gauge. We point out, however, that the finite 2PI kernel arises as the sum of the two singular pieces in Eqs. (13),(16). This is again a finding that will recur at NLO: the full discontinuity (4) of the gluon propagator in the ML prescription has a much milder behavior than the individual contributions to it.

It is instructive to contrast the result in Eq. (17) with the one obtained for the PV prescription [9]:

$$
\begin{aligned}
\Gamma_{q q}^{(a), P V}(x)= & \frac{\alpha_{s}}{2 \pi} \operatorname{PP} \int_{0}^{Q^{2}} d\left|k^{2}\right|\left|k^{2}\right|^{-1-\epsilon} \\
& \times C_{F}\left[\frac{1+x^{2}}{(1-x)_{+}}+2 I_{0} \delta(1-x)\right],
\end{aligned}
$$

where

$$
I_{0} \equiv \int_{0}^{1} \frac{u}{u^{2}+\delta^{2}} d u \approx-\ln \delta .
$$

Thus, the 2PI kernel for the PV prescription has a divergent coefficient of $\delta(1-x)$, resulting from the gauge denominator $1 / n l$ and being regularized by the parameter $\delta$.
The calculation of the LO splitting function is completed by determining the end point contributions at $x=1$, corresponding to $Z_{q}$ in Eq. (5) and given by the graphs in Fig. 1(b). They can be straightforwardly obtained ${ }^{1}$ using the UVsingular structure of the one-loop quark self-energy, determined for the ML prescription in [18]. One finds [7]

$$
Z_{q}=1-\frac{\alpha_{s}}{2 \pi} \frac{1}{\epsilon} C_{F} \frac{3}{2}, \quad \text { that is, } \quad \xi_{q}^{(0)}=\frac{3}{2} C_{F} .
$$

Putting everything together, one eventually obtains

$$
P_{q q}^{(0)}(x)=C_{F}\left[\frac{1+x^{2}}{(1-x)_{+}}+\frac{3}{2} \delta(1-x)\right],
$$

in agreement with [13]. We finally note that of course the same final answer is obtained within the PV prescription: the singular integral $I_{0}$ in Eq. (18) is cancelled by the contribution from $Z_{q}$, since we have [9]

$$
Z_{q}^{P V}=1-\frac{\alpha_{s}}{2 \pi} \frac{1}{\epsilon} C_{F}\left[\frac{3}{2}-2 I_{0}\right] .
$$

Thus, to summarize, the advantage of the ML prescription at the LO level mainly amounts to producing truly finite results for the 2PI kernels, as required for the method of $[11,9,10]$. Furthermore, there is no need for introducing renormalization constants depending on additional singular quantities like $I_{0}$ that represent a mix-up in the treatment of UV and IR singularities.

\section{CALCULATION OF THE FLAVOR NONSINGLET SPLITTING FUNCTION AT NLO}

At NLO, there are two different nonsinglet evolution kernels $P^{-,(1)}$ and $P^{+,(1)}$ governing the evolutions of the quark density combinations $q-\bar{q}$ and $q+\bar{q}-\left(q^{\prime}+\bar{q}^{\prime}\right)$, respectively. The two kernels are given in terms of the (flavordiagonal) quark-to-quark and quark-to-antiquark splitting functions by (see, for instance, Ref. [12])

$$
P^{ \pm,(1)} \equiv P_{q q}^{V,(1)} \pm P_{q \bar{q}}^{V,(1)},
$$

where the last splitting function originates from a tree graph that does not comprise any real-gluon emission and is therefore free of any problems related to the use of the light-cone gauge. Thus, we do not need to recalculate $P_{q \bar{q}}^{V,(1)}$. The Feynman diagrams contributing to $P_{q q}^{V,(1)}$ are collected in Fig. 2. We have labelled the graphs according to the notation of $[9,12]$. We also show the graphs contributing to $Z_{q}$ at two loops. We will not calculate all of these, since this is not really required. Their role will be discussed in Sec. III D.

\footnotetext{
${ }^{1}$ Alternatively one can obtain the contributions from the requirement $\int_{0}^{1} P_{q q}^{(0)}(x) d x=0[13,9]$.
} 

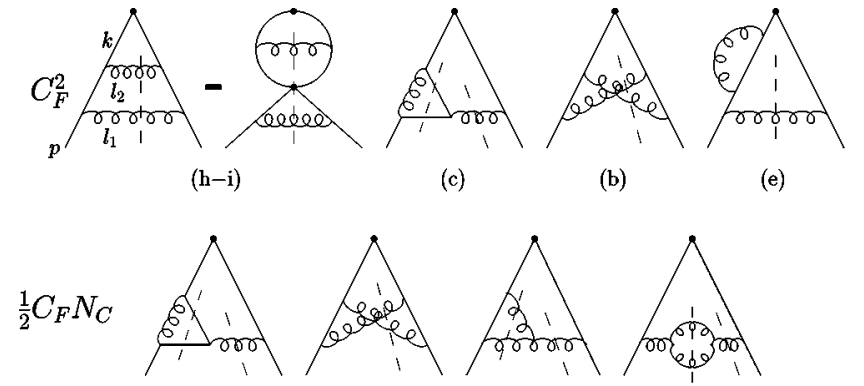

(c)

(b)

(d)

(f)

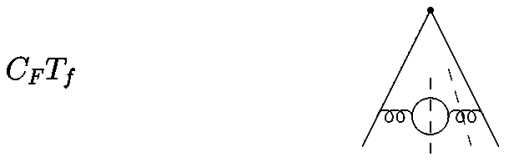

(g)
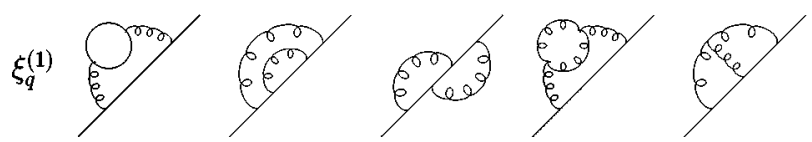

FIG. 2. Diagrams contributing to $\Gamma_{q q}$ at NLO.

\section{A. Virtual-cut diagrams and renormalization}

Many of the diagrams in Fig. 2 have real and virtual cuts, as has been indicated by the dashed lines. Let us start by discussing the contributions from the virtual cuts in graphs (c),(d),(e),(f),(g). It is clear that these essentially have the LO topology in the sense that there is always one outgoing gluon (momentum $l$ ), to be treated according to the ML prescription as discussed in the previous section. We recall that this means that there are two contributions for this gluon, one at $l^{2}=0$ and the other with $l^{2}+l_{\perp}^{2}=0$, corresponding to the gluon acting as an axial ghost. ${ }^{2}$ This immediately implies that we will have to calculate the loop integrals for these two situations. In addition, it is clear that the ML prescription also has to be used in the calculation of the loop itself, not just for the treatment of the external gluon. For instance, the gauge denominator $1 /(r \cdot n)$, where $r$ is the loop momentum, is subject to the prescription (1). In short, we will need several two-point and three-point functions with and without gauge denominators like $1 /(n \cdot r)$, and for both $l^{2}=0$ and $l^{2}+l_{\perp}^{2}=0$.

We point out that important qualitative differences with respect to the PV prescription arise here: in the PV calculation one always has $l^{2}=0$ for the outgoing gluon in the virtual-cut graphs, and there is no explicit dependence on $l_{\perp}^{2}$. For instance, the way to deal with the self-energies in graphs (f),(g) in the PV prescription is identical to their treatment in covariant gauges: one calculates them for off-shell $l^{2}$, renor-

\footnotetext{
${ }^{2}$ In the next subsection we will see that for diagrams (d),(f) there are also other contributions at $l^{2}+l_{\perp}^{2}=0$, not just the one from the axial ghost going into the loop. However, the integration of those contributions proceeds in exactly the same way as outlined here. We postpone the discussion of all the contributions at $l^{2}+l_{\perp}^{2}=0$ for graphs (d),(f) to the next subsection.
}

malizes them, and eventually takes the limit $l^{2} \rightarrow 0$. In this way, almost all contributions of the diagrams will vanish since all loop integrals have to be proportional to $\left(l^{2}\right)^{-\epsilon}(\epsilon$ $<0$ ) on dimensional grounds. Only the contribution from the $\overline{\mathrm{MS}}$ counterterm remains [19,9] because this is the only quantity not proportional to $\left(l^{2}\right)^{-\epsilon}$. In contrast to this, in the ML prescription $l_{\perp}^{2}$ sets an extra mass scale. For graph (f), one therefore encounters terms $\sim\left(l^{2}\right)^{-\epsilon}$, but also terms of the form $\sim\left(a l^{2}+b l_{\perp}^{2}\right)^{-\epsilon}$. The latter terms yield nonvanishing contributions to the virtual-cut result even at $l^{2}=0$. This is still not the case for graph $(\mathrm{g})$ since here the pure quark loop of course does not contain any light-cone gauge denominator and thus does not depend on $l_{\perp}^{2}$. Nevertheless, one gets a contribution from the quark loop in $(\mathrm{g})$ for $l^{2}$ $+l_{\perp}^{2}=0$, i.e., when the gluon running into the loop is an axial ghost, corresponding to the second part of the ML discontinuity in Eq. (4).

As expected, in the actual derivation of the loop integrals the property of the ML prescription to allow a Wick rotation is of great help. Nevertheless, some of the integrals are quite involved, since the ML prescription introduces an explicit dependence of the loop integrands on the transverse components $r_{\perp}^{2}$ due to the identity $2(n r)\left(n^{*} r\right)=r^{2}+r_{\perp}^{2}$. Furthermore, since we are interested in calculating also the contributions at $x=1$, we need to calculate the loop integrals up to $\mathcal{O}(\epsilon)$ rather than $\mathcal{O}(1)$. The reason for this is that very often the final answer for a loop calculation with $l^{2}=0$ will contain terms of the form $(1-x)^{-1-a \epsilon}(a=1,2)$, to be expanded according to Eq. (12). As a result, a further pole factor $1 / \epsilon$ is introduced into the calculation, yielding finite contributions when multiplied by the $\mathcal{O}(\epsilon)$ terms in the loop integrals. A similar thing happens in the loop part with $l^{2}$ $+l_{\perp}^{2}=0$. Here, an extra factor $1 / \epsilon$ can be introduced when integrating this part over the phase space in Eq. (15). The higher pole terms created in these ways will cancel out eventually, but not the finite parts they have generated in intermediate steps of the calculation. The detailed expressions for the loop integrals in the ML prescription are given in Appen$\operatorname{dix}$ A.

For the renormalization of the loop diagrams, one needs to subtract their UV poles, which is achieved in the easiest way by inserting the UV-divergent one-loop structures as calculated for the light-cone gauge in the ML prescription in $[6,18,20,21]$. All structures have also been compiled in [2]. The ones we need for the nonsinglet calculation are displayed in Fig. 3. One notices that, as expected, the structures are gauge dependent and Lorentz noncovariant. Even more, the expressions for the non-Abelian quantities $\Pi_{\mu \nu}^{g}$ and $\Gamma_{\mu}^{g}$ in Fig. 3 are nonpolynomial in the external momenta, owing to terms $\sim 1 /[n l]$. It is an important feature of the ML prescription that these nonlocal terms exist, but decouple from physical Green's functions [4] thanks to the orthogonality of the free propagator with respect to the gauge vector, $n_{\mu} \mathcal{D}^{\mu \nu}(l)=0$ (this has actually been an important ingredient for the proof [4] of the renormalizability of QCD in the ML light-cone gauge). Thus, the nonlocal pole parts never appear in our calculation. This is in contrast to the PV prescription, where one has [9] contributions from the renormalization 

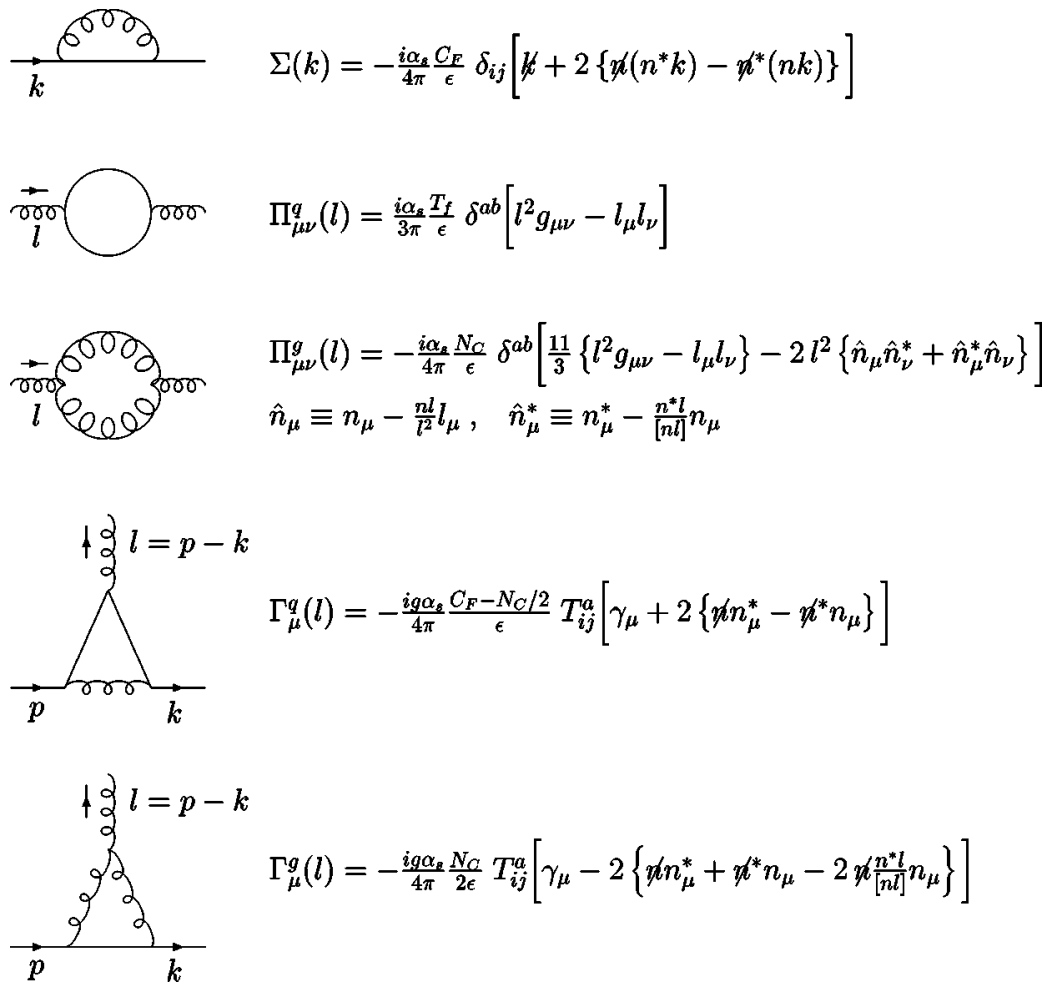

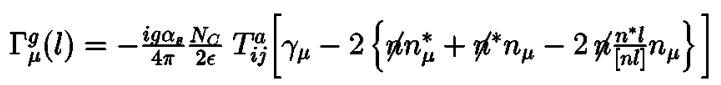

FIG. 3. UV-divergent one-loop structures as obtained in the light-cone gauge, using the ML prescription. The indices $i, j(a, b)$ denote quark (gluon) colors; $T^{a}$ are the generators of SU(3).

constants to the calculation that explicitly depend on the external momentum fractions $x, 1-x$.

\section{B. Real-cut graphs}

Let us now deal with the real cuts. One way of evaluating these is to integrate over the phase space of the two outgoing particles with momenta $l_{1}, l_{2}$ (for the notation of the momenta, see Fig. 2), in addition to the integration over the "observed" parton with momentum $k$. This is the strategy we have adopted for all diagrams contributing to the $C_{F}^{2}$ and the $C_{F} T_{f}$ parts of the splitting function, i.e., graphs (b),(c),(g),(h). For graphs (d),(f), we found it simpler to use a different method, as will be pointed out below.

If the two outgoing partons are gluons, their phase space in the ML prescription splits up into four pieces, as we discussed in Sec. I. It is possible to write down a phase space that deals with all four parts. We leave the details for Appendix B.

Upon integration of the squared real-cut matrix element for a diagram, each of the four parts of phase space gives highly divergent results, but their sum is usually less singular. This is similar to the pattern we found at LO. For instance, the phase space integration of graph (b) of Fig. 2 (before performing the final integration over $\left|k^{2}\right|$ ) is expected to give a finite result, since the graph is $2 \mathrm{PI}$ and possesses no virtual cut. This indeed turns out to be the case, but the individual contributions to (b) by the four parts of phase space all have poles $\sim 1 / \epsilon^{2}$ and $\sim 1 / \epsilon$ which cancel out when combined. A similar cancellation of higher pole terms happens for graph (h) [which of course is not finite by itself, but has a leftover 1/ $\epsilon$ singularity, to be cancelled by the contribution from diagram (i)]. Here even poles $\sim 1 / \epsilon^{3}$ occur at intermediate stages of the calculation. For those graphs that also have virtual cuts, the situation is in general even more complicated, as cancellations will occur only in the sum of the real and virtual cuts. An example for this case will be given in Sec. III C.

For graphs (d),(f), the phase space integrals become extremely complicated. This is due to the extra denominator $1 /\left(l_{1}+l_{2}\right)^{2}$ present in these graphs, which causes great complications in the axial-ghost parts of the phase space. For (f), we found it still possible to get the correct result via the " "phase space method," but for (d) this seemed a forbidding task. It turned out to be more convenient to determine the result in a different way: if one calculates, for instance, the gluon loop in graph (f) for an arbitrary off-shell momentum $l$ going into the loop, the imaginary part of the loop will correspond to the real-cut contribution we are looking for. To be more precise, the strategy goes as follows: we calculate the loop graph for off-shell $l$ and insert the result into the appropriate LO phase space. The latter can be derived as in Eq. (9), omitting, however, the $\delta\left(l^{2}\right)$ there. One finds

$$
\begin{aligned}
& x \int d^{d} k \int d^{d} l \delta\left(x-\frac{k n}{p n}\right) \delta(p-k-l) \\
& =\frac{\pi^{1-\epsilon}}{2 \Gamma(1-\epsilon)} \int_{0}^{Q^{2}} d\left|k^{2}\right| \int_{0}^{1 /(1-x)} d \tau\left(k_{\perp}^{2}\right)^{-\epsilon}
\end{aligned}
$$

where now 


$$
k_{\perp}^{2}=l_{\perp}^{2}=\left|k^{2}\right|(1-x) \tau, \quad l^{2}=\frac{\left|k^{2}\right|(1-x)}{x}(1-\tau) .
$$

The limits for the $\tau$ integration in Eq. (24) span the largest possible range for $\tau$, given by the conditions $l_{\perp}^{2}>0, l^{2}+l_{\perp}^{2}$ $>0$. The full imaginary part arising when performing the loop and the $\tau$ integrations has to correspond to the sum over all cuts in the diagram. One encounters discontinuities from the following sources.

(A) From the loop integrations. Here imaginary parts arise, for instance, if for certain values of $\tau$ and of the Feynman parameters $t_{1}, \ldots, t_{k}$, one finds terms of the form

$$
\left[f\left(t_{1}, \ldots, t_{k}, \tau\right)\right]^{-\epsilon}
$$

where $f$ is negative. Details for integrals with such properties are given in Appendix C. The imaginary part originating in this way essentially corresponds to the cut through the loop itself, i.e., to the real-cut contribution we are looking for.

(B) From the propagator $1 /\left(l^{2}+i \varepsilon\right)$ via the identity

$$
\frac{1}{l^{2}+i \varepsilon}=\mathrm{PV}\left(\frac{1}{l^{2}}\right)-i \pi \delta\left(l^{2}\right),
$$

where PV denotes the principal value. The imaginary part $\sim \delta\left(l^{2}\right)$ obviously represents the loop contribution at $l^{2}=0$ which we have determined in the last subsection. Therefore, we do not need to reconsider this part of the discontinuity.

(C) From terms $\sim 1 /[n l]$, for which a relation similar to Eq. (27) holds:

$$
\frac{1}{[n l]} \equiv \frac{1}{n l+i \varepsilon \operatorname{sgn}\left(n^{*} l\right)}=\mathrm{PV}\left(\frac{1}{n l}\right)-i \pi \operatorname{sgn}\left(n^{*} l\right) \delta(n l) .
$$

At first sight, one might think that the discontinuity $\sim \delta(n l)$ simply corresponds to the calculation of the gluon loop for the case when the gluon entering the loop is an axial ghost with $l^{2}=-l_{\perp}^{2}$. However, the situation is more subtle: The terms $\sim 1 /[n l]$ do not only originate from the propagators of the external gluons, but also from splitting formulas such as [2]

$$
\frac{1}{[n r][n(l-r)]}=\frac{1}{[n l]}\left(\frac{1}{[n r]}+\frac{1}{[n(l-r)]}\right)
$$

(where $r$ is the loop momentum), as well as from the loop integrals themselves, like in the case of

$$
\int \frac{d^{d} r}{(2 \pi)^{d}} \frac{1}{\left(r^{2}+i \varepsilon\right)\left[(l-r)^{2}+i \varepsilon\right][n r]} .
$$

All these terms $\sim 1 /[n l]$ have to be treated according to the ML prescription, i.e., give rise to discontinuities $\sim \delta(n l)$ $\sim \delta(1-x)$ via Eq. (28). The sum of all discontinuities arising in this way actually has to correspond to the "pure-axialghost" part of the graph, given by (a) the virtual-cut contribution when the gluon going into the loop is an axial ghost, plus (b) the real-cut contribution when both final-state gluons act as axial ghosts. These two parts cannot easily be separated from each other, which is the reason why we postponed the whole treatment of graphs (d),(f) at $l^{2}=-l_{\perp}^{2}$ to this section. The integrals needed to obtain this part of the discontinuity are those already mentioned in the last subsection and collected in the right-hand column of Table IV in Appendix A.

It is also worth mentioning that despite the fact that graph (f) has a squared gluon propagator, there are cancellations coming from the algebra in the numerator; as a consequence, one never encounters expressions like $1 /[n l]^{2}$ or $1 /\left(l^{2}\right.$ $+i \varepsilon)^{2}$ before taking the discontinuity, and Eqs. (28), (27) are all we need.

Clearly, when finally collecting all the imaginary parts from (A) and (C), the PV parts in (B) and (C) play a role in the calculation. While $1 / n l \sim 1 /(1-x)$ in Eq. (28) only diverges at the end point at $x=1$ where it is always regularized by factors such as $(1-x)^{-\epsilon}$, the propagator $1 / l^{2}$ in Eq. (27) in general has its singularity inside the region of the $\tau$ integration: from Eq. (25) one finds that $l^{2}>0$ for $\tau<1$, but $l^{2}<0$ for $\tau>1$. The principal-value prescription ${ }^{3}$ in Eq. (27) takes care of the pole at $\tau=1$ and leads to a cancellation of the positive spike for $\tau \rightarrow 1^{-}$and the negative one for $\tau$ $\rightarrow 1^{+}$, resulting in a perfectly well-defined finite result.

The vertex graph (d) can be treated in a similar fashion as (f). Here one calculates the full vertex for $p^{2}=0, k^{2}<0$, but arbitrary $l^{2}$, and determines the imaginary parts arising with respect to $l^{2}$. This corresponds again to point (A) above, and calculational details are also given in Appendix C. The imaginary part from (B) is again related to the virtual-cut contribution at $l^{2}=0$ that we already calculated in the last subsection. The discontinuity from (C) needs to be taken into account, and as before it corresponds to the full "pureghost" contribution (virtual cut and real cut), residing at $x$ $=1$.

A final comment concerns graph (i). Its contribution to $\Gamma_{q q}$ is essentially given by a convolution of two LO expressions, each corresponding to Fig. 1(a), keeping, however, also all finite terms in the upper part of the diagram, including the factor $(1-x)^{-\epsilon}$ from phase space [see Eqs. (11), (12)]:

$$
\text { (i) } \begin{aligned}
& \sim \frac{1}{\epsilon}\left[\frac{1+z^{2}}{(1-z)_{+}}-\epsilon\left(1+z^{2}\right)\left(\frac{\ln (1-z)}{1-z}\right)_{+}-\epsilon(1-z)\right] \\
& \otimes \frac{1}{\epsilon}\left[\frac{1+z^{2}}{(1-z)_{+}}\right],
\end{aligned}
$$

where

$$
(f \otimes g)(x) \equiv \int_{x}^{1} \frac{d z}{z} f\left(\frac{x}{z}\right) g(z) .
$$

Note that this is in contrast with the PV prescription where the contribution from (i) does not correspond to a

\footnotetext{
${ }^{3}$ To avoid confusion, we emphasize at this point that the principal value for $1 / l^{2}$ in Eq. (27) is well defined here and not related to the principal-value prescription for the light-cone denominator $1 / n l$ that we heavily criticized in the Introduction.
} 
genuine convolution in the mathematical sense. Since both convoluted functions in Eq. (31) contain distributions, the convolution itself will also be a distribution. The evaluation of Eq. (31) is most conveniently performed in Mellinmoment space where convolutions become simple products. Some details of the calculation and a few nonstandard moment expressions are given in Appendix D.

\section{Final results}

We now combine the results of the previous subsections. The first observation is that for the ML prescription all 2PI graphs, and also the difference $(h)-(i)$, turn out to give truly finite contributions to $\Gamma_{q q}$, before the final integration over $\left|k^{2}\right|$ is performed. This expectation for the light-cone gauge [11] was not really fulfilled by the PV prescription, where the results for the diagrams depended on integrals such as $I_{0}$ in Eq. (19) that diverge if the regularization $\delta$ of the PV prescription is sent to zero $[9,12]$. The finiteness of the kernels in the ML prescription comes about via delicate cancellations of terms sometimes as singular as $1 / \epsilon^{2}$ or even $1 / \epsilon^{3}$ when the various real-cut (gluon and axial-ghost) and virtual-cut contributions are added. To give just one example beyond those already discussed in the previous subsection, let us discuss the contributions of graph $(\mathrm{g})$ to $\Gamma_{q q}$. From the real-cut diagram, one has, up to trivial factors,

$$
\begin{aligned}
\Gamma_{q q}^{(\mathrm{g}), r} \sim & \left(\frac{\alpha_{s}}{2 \pi}\right)^{2} \operatorname{PP} \int_{0}^{Q^{2}} \frac{d\left|k^{2}\right|\left|k^{2}\right|^{-1-2 \epsilon}}{\Gamma(1-2 \epsilon)}\left[\delta(1-x)\left(\frac{2}{3 \epsilon^{2}}+\frac{10}{9 \epsilon}-\frac{2}{3} \zeta(2)+\frac{56}{27}\right)\right. \\
& \left.+\frac{1+x^{2}}{(1-x)_{+}}\left(-\frac{2}{3 \epsilon}-\frac{10}{9}\right)+\frac{4}{3}\left(1+x^{2}\right)\left(\frac{\ln (1-x)}{1-x}\right)_{+}-\frac{2}{3} \frac{1+x^{2}}{1-x} \ln x\right] .
\end{aligned}
$$

The virtual-cut graph for $l^{2}=-l_{\perp}^{2}$ (corresponding to the gluon being an axial ghost) contributes before renormalization:

$$
\Gamma_{q q}^{(\mathrm{g}), v} \sim\left(\frac{\alpha_{s}}{2 \pi}\right)^{2} \delta(1-x) \mathrm{PP} \int_{0}^{Q^{2}} \frac{d\left|k^{2}\right|\left|k^{2}\right|^{-1-2 \epsilon}}{\Gamma(1-2 \epsilon)}\left(-\frac{2}{3 \epsilon^{2}}-\frac{10}{9 \epsilon}-\frac{2}{3} \zeta(2)-\frac{56}{27}\right) .
$$

The loop with $l^{2}=0$ only contributes via its renormalization counterterm as explained earlier. This contribution exists also for the loop with $l^{2}=-l_{\perp}^{2}$ and reads, on aggregate for both loop parts,

$$
\Gamma_{q q}^{(\mathrm{g}), " \text { "ren" }} \sim\left(\frac{\alpha_{s}}{2 \pi}\right)^{2} \operatorname{PP} \int_{0}^{Q^{2}} \frac{d\left|k^{2}\right|\left|k^{2}\right|^{-1-\epsilon}}{\Gamma(1-2 \epsilon)} \frac{2}{3}\left[\frac{1}{\epsilon} \frac{1+x^{2}}{(1-x)_{+}}-\left(1+x^{2}\right)\left(\frac{\ln (1-x)}{1-x}\right)_{+}-1+x\right] .
$$

When adding the integrands of Eqs. (33)-(35), all poles cancel, and as promised the contribution to $\Gamma_{q q}$ is finite before integration over $\left|k^{2}\right|$.

Next, we determine the contributions of the various graphs to $P_{q q}^{V,(1)}$, making use of Eq. (7). The results are displayed in Tables I and II. We see that all entries in the tables are completely well defined, even at $x=1$, in terms of distributions, which is a property that we already encountered at LO.

The sums of the various graph-by-graph contributions are also presented in Tables I and II. One realizes that many more complicated structures, such as the dilogarithm $\mathrm{Li}_{2}(x)$, cancel in the sums. Considering only $x<1$ for the moment, it is the most important finding of this work that the entries in the columns "Sum' in Tables I and II exactly reproduce the results found in the PV calculations $[9,12]$ for $x<1$. Since the latter are in agreement with those obtained in the covariant-gauge OPE calculations [22], we conclude that the ML prescription has led to the correct final result. To a cer- tain extent, this is a check on the prescription itself in the framework of a highly nontrivial application. Since-in contrast to the PV recipe-the ML prescription possesses a solid field-theoretical foundation $[3,4]$, our calculation has finally provided a "clean" derivation of the NLO flavor nonsinglet splitting function within the CFP method, highlighting the viability of that method.

The next subsection will address the end point $[\delta(1$ $-x)]$ contributions to the NLO flavor nonsinglet splitting function.

\section{Contributions at $x=1$ and a sample two-loop calculation of $Z_{q}$}

In the PV calculations $[9,10,12]$ of the two-loop splitting functions the contributions $\sim \delta(1-x)$ were never directly calculated, but inferred $[9,23,12]$ from fermion number conservation, expressed by the requirement

$$
\int_{0}^{1}\left[P_{q q}^{V,(1)}(x)-P_{q \bar{q}}^{V,(1)}(x)\right] d x=0 .
$$


TABLE I. Final results for the $C_{F}^{2}$ part of $P_{q q}^{V,(1)}$ on a graph-by-graph basis.

\begin{tabular}{|c|c|c|c|c|c|}
\hline \multirow[b]{2}{*}{ Terms } & \multicolumn{5}{|c|}{$C_{F}^{2}$} \\
\hline & (b) & (c) & (e) & $(\mathrm{h}-\mathrm{i})$ & Sum \\
\hline$\delta(1-x)$ & $-2 \zeta(2)-2 \zeta(3)$ & $-3-\zeta(2)+12 \zeta(3)$ & $4-2 \zeta(2)-8 \zeta(3)$ & $2 \zeta(2)+4 \zeta(3)$ & $1-3 \zeta(2)+6 \zeta(3)$ \\
\hline$\frac{1+x^{2}}{(1-x)_{+}}$ & & $-7+4 \zeta(2)$ & $7-4 \zeta(2)$ & & \\
\hline$\left(1+x^{2}\right)\left(\frac{\ln (1-x)}{1-x}\right)_{+}$ & 4 & -3 & 3 & -4 & \\
\hline$\frac{1+x^{2}}{1-x} \ln x \ln (1-x)$ & -2 & 4 & -4 & & -2 \\
\hline$\frac{1+x^{2}}{1-x} \ln ^{2} x$ & & -1 & & 1 & \\
\hline$\frac{1+x^{2}}{1-x} \ln x$ & -1 & $-\frac{1}{2}$ & -1 & 1 & $-\frac{3}{2}$ \\
\hline$\frac{1+x^{2}}{1-x}\left[\operatorname{Li}_{2}(x)-\zeta(2)\right]$ & -2 & 4 & -4 & 2 & \\
\hline$(1+x) \ln ^{2} x$ & & & & $-\frac{1}{2}$ & $-\frac{1}{2}$ \\
\hline $\ln (1-x)$ & & 2 & -2 & & \\
\hline$x \ln x$ & 1 & $-\frac{5}{2}$ & -1 & -1 & $-\frac{7}{2}$ \\
\hline $\ln x$ & 1 & $-\frac{9}{2}$ & 1 & 1 & $-\frac{3}{2}$ \\
\hline$x$ & & 9 & -1 & -3 & 5 \\
\hline 1 & & -5 & -3 & 3 & -5 \\
\hline
\end{tabular}

We could proceed in the same way and stop the calculation here. However, the calculation we have performed in the ML prescription allows us to go beyond this pragmatic approach, since-at least for the $C_{F}^{2}$ and $C_{F} T_{f}$ parts-we have always picked up the finite amounts of $\delta(1-x)$ contributed by the 2PI kernels. If we now performed a calculation of $\xi_{q}^{(1)}$, corresponding to the graphs in the bottom row of Fig. 2, we would have all terms $\sim \delta(1-x)$ in the NLO flavor nonsinglet splitting function and could check whether indeed Eq. (36) is correctly reproduced. As an example, we will go this way for the $C_{F} T_{f}$ part of the splitting function.

Let us first establish what we need to get for $\xi_{q}^{(1)}$. The coefficient of $\delta(1-x)$ in the NLO splitting function was determined in $[23,12]$ via Eq. (36) to be

$$
\begin{aligned}
& C_{F}^{2}\left(\frac{3}{8}-3 \zeta(2)+6 \zeta(3)\right)+C_{F} T_{f}\left(-\frac{1}{6}-\frac{4}{3} \zeta(2)\right) \\
& \quad+C_{F} N_{C}\left(\frac{17}{24}+\frac{11}{3} \zeta(2)-3 \zeta(3)\right),
\end{aligned}
$$

while in our calculation we have, according to Eq. (8) and Tables I and II,

$$
\begin{aligned}
\xi_{q}^{(1)} & +C_{F}^{2}[1-3 \zeta(2)+6 \zeta(3)] \\
& +C_{F} T_{f}\left(-\frac{4}{3} \zeta(2)\right)+C_{F} N_{C}\left(\frac{11}{3} \zeta(2)+\ldots\right),
\end{aligned}
$$

where the ellipsis indicates that we have not entirely calculated the finite amount of $\delta(1-x)$ in the $C_{F} N_{C}$ part of $P_{q q}^{V,(1)}$, even though we were able to determine its contribution $\sim \zeta(2)$ (see Appendix C). Comparing Eqs. (37) and (38), we get a prediction for the $C_{F}^{2}$ and $C_{F} T_{f}$ parts of $\xi_{q}^{(1)}$ in the light-cone gauge with the ML prescription:

$$
\xi_{q}^{(1)}=-\frac{5}{8} C_{F}^{2}-\frac{1}{6} C_{F} T_{f}+C_{F} N_{C}(\ldots)
$$

where all we can say about the $C_{F} N_{C}$ part is that it does not contain any terms $\sim \zeta(2)$. It is quite remarkable that no $\zeta(2)$, $\zeta(3)$ terms are left over in the $C_{F}^{2}$ and $C_{F} T_{f}$ parts of $\xi_{q}^{(1)}$.

To directly calculate the $C_{F} T_{f}$ part of $\xi_{q}^{(1)}$, we only have to evaluate the first diagram in the bottom row of Fig. 2. What we need to extract is the two-loop renormalization constant for that diagram, when the light-cone gauge with the ML prescription is used. The calculation is relatively easy 
TABLE II. Final results for the $C_{F} N_{C}$ and $C_{F} T_{f}$ parts of $P_{q q}^{V,(1)}$ on a graph-by-graph basis. The dots in the contributions $\sim \delta(1-x)$ in the first row denote uncalculated pieces consisting of $\zeta(3)$ and a rational number (see Appendix C).

\begin{tabular}{|c|c|c|c|c|c|c|}
\hline & & & $\frac{1}{2} C_{F} N_{C}$ & & & $C_{F} T_{f}$ \\
\hline Terms & (b) & (c) & (d) & (f) & Sum & (g) \\
\hline$\delta(1-x)$ & $2 \zeta(2)+2 \zeta(3)$ & $3+\zeta(2)-12 \zeta(3)$ & $5 \zeta(2)+\cdots$ & $-\frac{2}{3} \zeta(2)+\cdots$ & $\frac{22}{3} \zeta(2)+\cdots$ & $-\frac{4}{3} \zeta(2)$ \\
\hline$\frac{1+x^{2}}{(1-x)_{+}}$ & & $7-4 \zeta(2)$ & $-3+2 \zeta(2)$ & $\frac{31}{9}$ & $\frac{67}{9}-2 \zeta(2)$ & $-\frac{10}{9}$ \\
\hline$\left(1+x^{2}\right)\left(\frac{\ln (1-x)}{1-x}\right)_{+}$ & -4 & 3 & 1 & & & \\
\hline$\frac{1+x^{2}}{1-x} \ln x \ln (1-x)$ & 2 & -4 & 2 & & & \\
\hline$\frac{1+x^{2}}{1-x} \ln ^{2} x$ & & 1 & & & 1 & \\
\hline$\frac{1+x^{2}}{1-x} \ln x$ & 1 & $\frac{1}{2}$ & $\frac{5}{2}$ & $-\frac{1}{3}$ & $\frac{11}{3}$ & $-\frac{2}{3}$ \\
\hline$\frac{1+x^{2}}{1-x}\left[\operatorname{Li}_{2}(x)-\zeta(2)\right]$ & 2 & -4 & -2 & 4 & & \\
\hline $\ln (1-x)$ & & -2 & 6 & -4 & & \\
\hline$x \ln x$ & -1 & $\frac{5}{2}$ & $\frac{1}{2}$ & & 2 & \\
\hline $\ln x$ & -1 & $\frac{9}{2}$ & $-\frac{11}{2}$ & 4 & 2 & \\
\hline$x$ & & -9 & -1 & $-\frac{10}{3}$ & $-\frac{40}{3}$ & $\frac{4}{3}$ \\
\hline 1 & & 5 & -3 & $\frac{34}{3}$ & $\frac{40}{3}$ & $-\frac{4}{3}$ \\
\hline
\end{tabular}

since the inner quark loop has obviously no light-cone gauge propagator and can in fact be calculated exactly:

$\Pi_{\mu \nu}(r)=-i T_{f} \frac{\alpha_{s}}{4 \pi} \frac{8 \Gamma^{2}(2-\epsilon) \Gamma(\epsilon)}{\Gamma(4-2 \epsilon)}\left(\frac{4 \pi}{-r^{2}}\right)^{\epsilon}\left[r^{2} g_{\mu \nu}-r_{\mu} r_{\nu}\right]$.

This self-energy can then be renormalized with the help of the counterterm in Fig. 3. The renormalized loop is then inserted into the outer loop. Here it is very convenient that $\Pi_{\mu \nu}$ is transverse:

$$
\mathcal{D}^{\alpha \mu}(r)\left[r^{2} g_{\mu \nu}-r_{\mu} r_{\nu}\right] \mathcal{D}^{\nu \beta}(r) \sim \mathcal{D}^{\alpha \beta}(r) .
$$

In other words, the whole calculation is not very different from a simple one-loop calculation of the quark self-energy, the only exception being that we now need loop integrals with the extra factor $\left(-r^{2}\right)^{-\epsilon}$ present in Eq. (40). If we embed the whole graph into the Dirac trace as shown in Fig. 1(b), it turns out that we only need a few integrals of this kind; they are collected in Appendix E. Since we have renormalized the inner loop, the leftover divergence after loop integration determines the two-loop counterterm and thus the contribution to $\xi_{q}^{(1)}$. We find, in the $\overline{\mathrm{MS}}$ scheme,

$$
Z_{q}^{C_{F} T_{f}}=1+\left(\frac{\alpha_{s}}{2 \pi}\right)^{2} C_{F} T_{f}\left(-\frac{1}{2 \epsilon^{2}}+\frac{1}{12 \epsilon}\right)
$$

Comparing to Eq. (8) this implies that the $C_{F} T_{f}$ part of $\xi_{q}^{(1)}$ is exactly what we expected it to be in Eq. (39):

$$
\xi_{q}^{(1), C_{F} T_{f}}=-\frac{1}{6} C_{F} T_{f} .
$$

This result clearly demonstrates the consistency of the whole approach: our example shows that the light-cone gauge method of $[11,9,10]$ is also able to determine the contributions $\sim \delta(1-x)$ to the splitting functions by explicit calculation. It would be interesting in this context to calculate also the other contributions to $\xi_{q}^{(1)}$ [and the missing part $\sim C_{F} N_{C} \delta(1-x)$ in our Table II]; important steps in this direction have been taken in [24,25] by examining the second and third diagrams in the bottom row of Fig. 2, which 
yield the $C_{F}^{2}$ part of $\xi_{q}^{(1)}$. Indeed it turns out that the results of $[24,25]$ can be exploited to reproduce the term $-5 C_{F}^{2} / 8$ in our prediction (39) for $\xi_{q}^{(1)}$, which can be regarded as a further confirmation of our results.

We have to admit, however, at this point that the ability to obtain the correct end point contributions is not restricted to the ML prescription: this is also possible for the PV prescription. Here, the coefficient of $\delta(1-x)$ in the $C_{F} T_{f}$ part of $P_{q q}^{V,(1)}$ reads

$$
\xi_{q, \mathrm{PV}}^{(1), C_{F} T_{f}}-C_{F} T_{f} \frac{20}{9} I_{0} .
$$

Here the second term originates from the entry " $-10 / 9$ " in the last column of Table II, when we omit the "plus" prescription there and reintroduce it using the PV identity [9]

$$
\frac{1}{1-x} \rightarrow I_{0} \delta(1-x)+\frac{1}{(1-x)_{+}},
$$

where $I_{0}$ is as defined in Eq. (19). Furthermore, $\xi_{q, \mathrm{PV}}^{(1), C_{F} T_{f}}$ denotes the $C_{F} T_{f}$ part of $\xi_{q}^{(1)}$ when the PV prescription is used. The explicit calculation gives

$$
\begin{aligned}
Z_{q, \mathrm{PV}}^{C_{F} T_{f}}= & 1+\left(\frac{\alpha_{s}}{2 \pi}\right)^{2} C_{F} T_{f}\left[-\frac{1}{2 \epsilon^{2}}\left(1-\frac{4}{3} I_{0}\right)\right. \\
& \left.+\frac{1}{\epsilon}\left(\frac{1}{12}+\frac{2}{3} \zeta(2)-\frac{10}{9} I_{0}\right)\right],
\end{aligned}
$$

that is,

$$
\xi_{q, \mathrm{PV}}^{(1), C_{F} T_{f}}=C_{F} T_{f}\left(-\frac{1}{6}-\frac{4}{3} \zeta(2)+\frac{20}{9} I_{0}\right) .
$$

It is interesting to see how upon combining Eqs. (44) and (47) the $I_{0}$ terms drop out, and the $C_{F} T_{f}$ part of the end point contributions comes out correctly as in Eq. (37) also for the PV prescription. We note, however, that again this happens at the expense of having renormalization constants depending on singular quantities like $I_{0}$ that represent a mix-up in the treatment of UV and IR singularities.

\section{CALCUlation OF THE $N_{C}^{2}$ PART OF THE SINGLET SPLITTING FUNCTION $P_{g g}$ AT NLO}

Let us now turn to the calculation of $P_{g g}^{(1)}$. We restrict ourselves to its $N_{C}^{2}$ part, since the contributions $\sim C_{F} T_{f}$, $N_{C} T_{f}$ are essentially trivial as far as the treatment of the LCA gauge is concerned: The $C_{F} T_{f}$ part comprises no gluon emission at all, and all diagrams contributing to the $N_{C} T_{f}$ part contain a quark loop and the emission of at most one gluon. Such diagrams with one-gluon emission have the LO kinematics and will not reveal any new features as compared to what we have already discussed. In contrast to this, the $N_{C}^{2}$ part of $P_{g g}^{(1)}$ requires the renormalization of the non-Abelian part of the three-gluon vertex and therefore really provides a further challenge for the ML prescription.

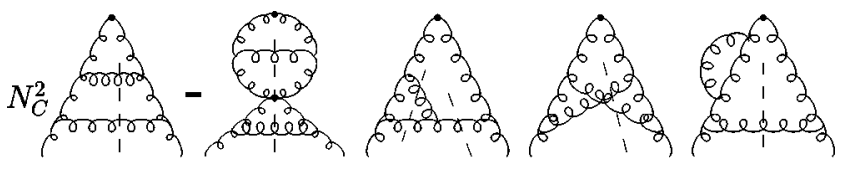

$(\mathrm{h}-\mathrm{i})$

(d)

(b)

(e)

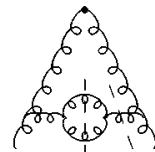

(f)

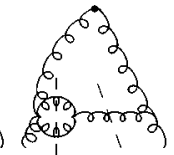

(s1)

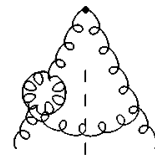

(s2)

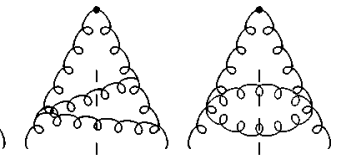

(j)

(k)
FIG. 4. Diagrams contributing to the $N_{C}^{2}$ part of $\Gamma_{g g}$ at NLO.

The diagrams contributing to the $N_{C}^{2}$ part of $\Gamma_{g g}$ at NLO are shown in Fig. 4. We do not show here the graphs contributing to $Z_{g}$ at two loops, since we will not attempt to calculate them.

The calculation of the various real-cut and virtual-cut diagrams proceeds in exactly the same way as before. For the renormalization of the virtual-cut contributions in the triangle graph (d) and the "swordfish" ones (s1),(s2), we need the UV counterterm for the three-gluon vertex in the lightcone gauge with the ML prescription. Here we can rely on the result presented in [26] (see also [27,2]); the part of it that is relevant for our calculation is recalled in Fig. 5.

Concerning the real cuts, we mention that for graphs (h), (b), (j),(k) we use the expression in Eq. (B5) for the phase space. As for the $C_{F} N_{C}$ part of $P_{q q}^{V,(1)}$, we found it easier to determine the contributions of the real cuts of the remaining diagrams via the extraction of the imaginary parts of the associated virtual graphs.

We have verified that again for the ML prescription all 2PI graphs give truly finite contributions to $\Gamma_{g g}$, before the final integration over $\left|k^{2}\right|$ is performed. This also applies to the end point $x=1$, where the result for each graph is again always well defined in terms of distributions and, as before, also has a coefficient of $\delta(1-x)$ that contains no $1 / \epsilon$ poles. Table III presents the contributions of the various diagrams to $P_{g g}^{(1)}$. Here we have defined the functions

$$
\begin{aligned}
p_{g g}(x) & \equiv \frac{\left(1-x+x^{2}\right)^{2}}{x(1-x)_{+}}, \\
l_{g g}(x) & \equiv \frac{\left(1-x+x^{2}\right)^{2}}{x}\left(\frac{\ln (1-x)}{1-x}\right)_{+}, \\
S_{2}(x) & \equiv \int_{x /(1+x)}^{1 /(1+x)} \frac{d z}{z} \ln \left(\frac{1-z}{z}\right) \\
& =-2 \operatorname{Li}_{2}(-x)-2 \ln x \ln (1+x)+\frac{1}{2} \ln ^{2} x-\zeta(2) .
\end{aligned}
$$

We mention in passing that graph (j) and the "swordfish" diagram (s1) give vanishing contributions to $P_{g g}^{(1)}$ if the PV 

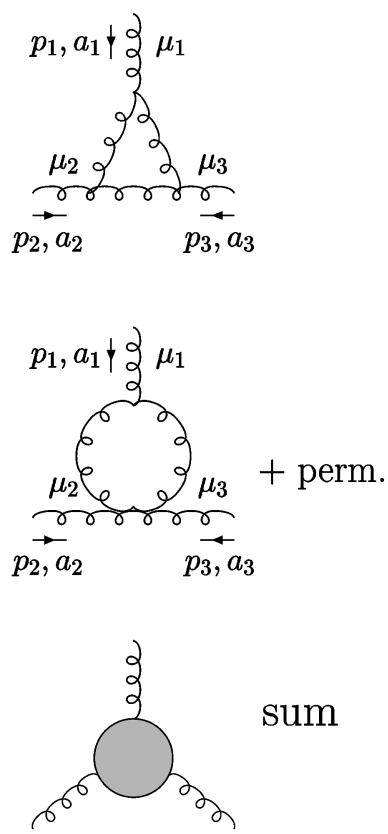

sum

$$
\begin{aligned}
T_{\mu_{1} \mu_{2} \mu_{3}} & \left(p_{1}, p_{2}, p_{3}\right)=\frac{i g \alpha_{s}}{4 \pi} \frac{N_{C}}{2 \epsilon} f^{a_{1} a_{2} a_{3}}\left[\frac{4}{3} A_{\mu_{1} \mu_{2} \mu_{3}}+2 C_{\mu_{1} \mu_{2} \mu_{3}}+\ldots\right] \\
A_{\mu_{1} \mu_{2} \mu_{3}}= & g_{\mu_{2} \mu_{3}}\left(p_{2}-p_{3}\right)_{\mu_{1}}+g_{\mu_{3} \mu_{1}}\left(p_{3}-p_{1}\right)_{\mu_{2}}+g_{\mu_{1} \mu_{2}}\left(p_{1}-p_{2}\right)_{\mu_{3}} \\
C_{\mu_{1} \mu_{2} \mu_{3}}= & g_{\mu_{2} \mu_{3}} n_{\mu_{1}}^{*}\left(p_{2}-p_{3}\right) \cdot n+g_{\mu_{3} \mu_{1}} n_{\mu_{2}}^{*}\left(p_{3}-p_{1}\right) \cdot n \\
& +g_{\mu_{1} \mu_{2}} n_{\mu_{3}}^{*}\left(p_{1}-p_{2}\right) \cdot n
\end{aligned}
$$

$$
S_{\mu_{1} \mu_{2} \mu_{3}}\left(p_{1}, p_{2}, p_{3}\right)=\frac{i g \alpha_{s}}{4 \pi} \frac{N_{C}}{2 \epsilon} f^{a_{1} a_{2} a_{3}}\left[6 A_{\mu_{1} \mu_{2} \mu_{3}}-6 C_{\mu_{1} \mu_{2} \mu_{3}}+\ldots\right]
$$

$$
\begin{aligned}
\Gamma_{\mu_{1} \mu_{2} \mu_{3}}\left(p_{1}, p_{2}, p_{3}\right) & =T_{\mu_{1} \mu_{2} \mu_{3}}\left(p_{1}, p_{2}, p_{3}\right)+S_{\mu_{1} \mu_{2} \mu_{3}}\left(p_{1}, p_{2}, p_{3}\right) \\
& =\frac{i g \alpha_{s}}{4 \pi} \frac{N_{C}}{\epsilon} f^{a_{1} a_{2} a_{3}}\left[\frac{11}{3} A_{\mu_{1} \mu_{2} \mu_{3}}-2 C_{\mu_{1} \mu_{2} \mu_{3}}+\ldots\right]
\end{aligned}
$$

FIG. 5. UV-divergent structures of the non-Abelian part of the three-gluon vertex as obtained in the light-cone gauge, using the ML prescription. $p_{1}, p_{2}, p_{3}$ denote the momenta of the external gluons, $a_{1}, a_{2}, a_{3}$ are the associated color indices $\left[f^{a_{1} a_{2} a_{3}}\right.$ being the structure constants of SU(3)], and $\mu_{1}, \mu_{2}, \mu_{3}$ are Lorentz indices. The dots indicate structures (some of them nonlocal) which do not contribute to our calculation thanks to the orthogonality of the free propagator to the gauge vector $n$.

prescription is used, but are nonvanishing for the ML prescription, where finite contributions arise from their ghost parts.

As for the case of $P_{q q}^{V,(1)}$, the full result for the $N_{C}^{2}$ part of $P_{g g}^{(1)}$, given by the column "Sum," is (at $\left.x<1\right)$ in agreement with the PV result of [10], which in turn coincides with the OPE one ${ }^{4}[15]$. Thus, the CFP method with ML prescription has also led to the correct final answer in this case, which clearly constitutes a further nontrivial and complementary check. As can be seen from Table III, we have not determined the finite amounts of contributions $\sim \delta(1-x)$ for the graphs since, like in the case of the $C_{F} N_{C}$ part of $P_{q q}^{V,(1)}$, these are quite hard to extract in some cases. The end point contributions to $P_{g g}^{(1)}$ can then only be derived from the energy-momentum conservation condition [23,12]. We emphasize, however, that, just as for $P_{q q}^{V,(1)}$, there is no principal problem concerning the calculation of the end point contributions: had we calculated the full $\delta(1-x)$ terms in Table III and the two-loop quantity $\xi_{g}^{(1)}$, all end point contributions would be at our disposal, and it would no longer be necessary to invoke the energy-momentum conservation condition; in fact, this could serve as a further check of the calculation.

\section{CONCLUSIONS}

We have performed a new evaluation of the NLO flavor nonsinglet splitting function and of the $N_{C}^{2}$ part of the NLO

\footnotetext{
${ }^{4}$ See also our discussion in the Introduction concerning the OPE calculations $[15,14]$ of $P_{g g}^{(1)}$.
}

gluon-to-gluon splitting function within the light-cone gauge method of $[11,9]$. The new feature of our calculation is the use of the Mandelstam-Leibbrandt prescription for dealing with the spurious poles generated by the gauge denominator in the gluon propagator. In contrast to the principal-value prescription employed in previous calculations $[9,10,12]$, the ML prescription has a solid field-theoretical foundation and will therefore provide a "cleaner" derivation of the result. As expected, the final results come out correctly, i.e., are in agreement with the ones in $[9,10,12,22]$. This finding is both a corroboration of the usefulness of the general method of $[11,9]$ to calculate splitting functions and a useful check on the ML prescription itself in a highly nontrivial application.

We have also discussed the $\delta(1-x)$ contributions to the NLO flavor nonsinglet splitting function, performing an explicit sample calculation of a two-loop contribution to the renormalization constant $Z_{q}$ in the ML light-cone gauge. It turns out that one indeed obtains the right amount of contributions at $x=1$ as required by fermion number conservation.

We conclude by conceding that the ML prescription is in general much more complicated to handle than the simpler, but less well-founded, PV prescription. With regard to future applications at, for instance, three-loop order, this creates a certain dilemma: the ML prescription might be too complicated to be used in that case, while on the other hand the ill-understood success of the PV prescription at the two-loop level does not guarantee that it will also produce correct results beyond.

\section{ACKNOWLEDGMENTS}

This work was supported in part by the EU Fourth Framework Program "Training and Mobility of Researchers,", 
TABLE III. Final results for the $N_{C}^{2}$ part of $P_{g g}^{(1)}$ on a graph-by-graph basis. The table does not include the coefficients of $\delta(1-x)$, which we have not determined. However, as mentioned in the main text, we have proved that each graph contributes a finite amount of $\delta(1-x)$ to $\Gamma_{g g}$ (before the final integration over $\left|k^{2}\right|$ is performed).

\begin{tabular}{|c|c|c|c|c|c|c|c|c|c|}
\hline \multirow[b]{2}{*}{ Terms } & \multicolumn{9}{|c|}{$N_{C}^{2}$} \\
\hline & (b) & (d) & $(\mathrm{s} 1+\mathrm{s} 2)$ & (e) & (f) & $(h-i)$ & (j) & $(\mathrm{k})$ & Sum \\
\hline$p_{g g}(x)$ & & $-\frac{98}{9}+6 \zeta(2)$ & & $\frac{134}{9}-8 \zeta(2)$ & $\frac{31}{9}$ & & & & $\frac{67}{9}-2 \zeta(2)$ \\
\hline$l_{g g}(x)$ & 4 & $-\frac{10}{3}$ & & $\frac{22}{3}$ & & -8 & & & \\
\hline$p_{g g}(x) \ln x \ln (1-x)$ & -2 & 6 & & -8 & & & & & -4 \\
\hline$p_{g g}(x) \ln ^{2} x$ & & -1 & & & & 2 & & & 1 \\
\hline$p_{g g}(x) \ln x$ & $-\frac{3}{2}$ & $\frac{11}{6}$ & $-\frac{3}{2}$ & -1 & $-\frac{1}{3}$ & 1 & $\frac{3}{2}$ & & \\
\hline$p_{g g}(x)\left[\mathrm{Li}_{2}(x)-\zeta(2)\right]$ & -2 & 2 & & -8 & 4 & 4 & & & \\
\hline$p_{g g}(-x) S_{2}(x)$ & 2 & & & & & & & & 2 \\
\hline$(1+x) \ln ^{2} x$ & & & & & & 4 & & & 4 \\
\hline$x^{2} \ln x$ & $-\frac{31}{6}$ & $\frac{11}{6}$ & $-\frac{3}{2}$ & -2 & -3 & $-\frac{19}{3}$ & $\frac{3}{2}$ & & $-\frac{44}{3}$ \\
\hline$x \ln x$ & -8 & $-\frac{52}{3}$ & 3 & 4 & 6 & 16 & & & $\frac{11}{3}$ \\
\hline $\ln x$ & $-\frac{17}{2}$ & $\frac{26}{3}$ & -3 & -5 & -5 & 3 & $\frac{3}{2}$ & & $-\frac{25}{3}$ \\
\hline$\frac{1}{x} \ln x$ & $-\frac{25}{6}$ & $-\frac{67}{6}$ & $\frac{3}{2}$ & 1 & 4 & $\frac{31}{3}$ & $-\frac{3}{2}$ & & \\
\hline$x^{2} \ln (1-x)$ & & -4 & & 1 & 3 & & & & \\
\hline$x \ln (1-x)$ & & 13 & -3 & -4 & -6 & & & & \\
\hline $\ln (1-x)$ & & $-\frac{25}{2}$ & $\frac{3}{2}$ & 6 & 5 & & & & \\
\hline$\frac{1}{x} \ln (1-x)$ & & 8 & & -4 & -4 & & & & \\
\hline$x^{2}$ & $\frac{136}{9}$ & $\frac{13}{3}$ & 9 & 1 & $-\frac{20}{3}$ & $-\frac{46}{3}$ & & & $\frac{67}{9}$ \\
\hline$x$ & $-\frac{105}{8}$ & $-\frac{16}{3}$ & -9 & -6 & $\frac{139}{12}$ & $\frac{19}{2}$ & & $-\frac{9}{8}$ & $-\frac{27}{2}$ \\
\hline 1 & $\frac{105}{8}$ & $\frac{59}{6}$ & $-\frac{9}{2}$ & 12 & $-\frac{103}{12}$ & $-\frac{19}{2}$ & & $\frac{9}{8}$ & $\frac{27}{2}$ \\
\hline$\frac{1}{x}$ & $-\frac{136}{9}$ & $-\frac{22}{3}$ & & -8 & $\frac{23}{3}$ & $\frac{46}{3}$ & & & $-\frac{67}{9}$ \\
\hline
\end{tabular}

Network "Quantum Chromodynamics and the Deep Structure of Elementary Particles,'” contract FMRX-CT98-0194 (DG 12-MIHT).

\section{APPENDIX A: VIRTUAL INTEGRALS}

Here we list some loop integrals needed for the calculation. We do not need to recall any of the covariant integrals, which are standard, but will only present those with a lightcone gauge denominator, to be treated according to the ML prescription (1).

We begin by performing a sample calculation of the integral

$$
\begin{aligned}
I(n, q) & \equiv \int \frac{d^{d} r}{(2 \pi)^{d}} \frac{1}{\left(r^{2}+i \varepsilon\right)\left[(q-r)^{2}+i \varepsilon\right][n r]} \\
& =\int \frac{d^{d} r}{(2 \pi)^{d}} \frac{n^{*} r}{\left(r^{2}+i \varepsilon\right)\left[(q-r)^{2}+i \varepsilon\right]\left(n r n^{*} r+i \varepsilon\right)} .
\end{aligned}
$$

We recall the definitions [9]

$$
n=\frac{p n}{2 P}(1,0, \ldots, 0,-1)
$$

$$
n^{*}=\frac{P}{p n}(1,0, \ldots, 0,1) \equiv \frac{1}{p n} p,
$$

where $p=P(1,0, \ldots, 0,1)$ is the momentum of the incoming quark: see Fig. 2. Introducing Feynman parameters, one has

$$
\begin{aligned}
I(n, q)= & \frac{4 P}{p n} \int_{0}^{1} d t \int_{0}^{1-t} d s \\
& \times \int \frac{d^{d} r}{(2 \pi)^{d}} \frac{r_{0}-r_{z}}{\left[r^{2}+s r_{\perp}^{2}-2(q \cdot r) t+q^{2} t+i \varepsilon\right]^{3}} .
\end{aligned}
$$

After performing a Wick rotation and straightforward integrations over $r$ one arrives at 


$$
\begin{aligned}
I(n, q)= & \frac{i \Gamma(1+\epsilon)}{16 \pi^{2}} \frac{2 n^{*} q}{q^{2}+i \varepsilon}\left(\frac{4 \pi}{-q^{2}}\right) \int_{0}^{1} d t t^{-\epsilon}(1-t)^{-1-\epsilon} \\
& \times \int_{0}^{1} d s\left(1+s t \frac{q^{2}+q_{\perp}^{2}+i \varepsilon}{\left(q^{2}+i \varepsilon\right)(1-t)}\right)^{-1-\epsilon}
\end{aligned}
$$

For example, for the case $q=k$ one finds

$$
\begin{aligned}
I(n, k)= & \frac{i \Gamma(1+\epsilon)}{16 \pi^{2}}\left(\frac{4 \pi}{\left|k^{2}\right|}\right)^{\epsilon} \frac{1}{[n k]} \\
& \times\left[\zeta(2)-\mathrm{Li}_{2}\left(\frac{k_{\perp}^{2}}{\left|k^{2}\right|}\right)+2 \epsilon \zeta(3)\right],
\end{aligned}
$$

where we have kept those terms that contribute to the final answer. In Eq. (A5), $\zeta(n)$ is Riemann's $\zeta$ function and $\mathrm{Li}_{2}(x)$ denotes the dilogarithm, defined by [28]

$$
\operatorname{Li}_{2}(z) \equiv-\int_{0}^{1} \frac{\ln (1-z y)}{y} d y .
$$

The result in Eq. (A5) coincides with the one in [2] for $\epsilon$ $=0$. Note that the ML prescription arising for $1 /[n k]$ is actually immaterial here since $n k=x p n$ never vanishes.

Setting, on the other hand, $q=l$ one gets, for $l^{2}=0$,

$$
I(n, l)=\frac{i \Gamma(1+\epsilon)}{16 \pi^{2}}\left(\frac{4 \pi}{-\left|k^{2}\right|(1-x)}\right)^{\epsilon} \frac{1}{n l} \frac{1}{2 \epsilon^{2}}
$$

and, for $l^{2}=-l_{\perp}^{2}$,

$$
I(n, l)=\frac{i \Gamma(1+\epsilon)}{16 \pi^{2}}\left(\frac{4 \pi}{-l^{2}}\right)^{\epsilon} \frac{2 n^{*} l}{l^{2}} B(-\epsilon, 1-\epsilon) .
$$

Note that the real part of Eq. (A7) has to be taken. ${ }^{5}$ Table IV contains all the required integrals with an ML light-cone gauge denominator. The integrals in the first column are for $l^{2}=0$; they depend on

$$
x=\frac{n k}{p n} \quad \text { and } \tilde{x}=\frac{n l}{p n}=1-x .
$$

Recall that terms $\sim(1-x)^{-1-a \epsilon}$ will lead to further poles, as was shown by Eq. (12). The integrals in the second column of Table IV are for the axial-ghost case, $l^{2}=-l_{\perp}^{2}$, and eventually need to be integrated further over the variable $\kappa$ defined in Eqs. (15),(14). The $\kappa$ integration produces further poles. We have

$$
\kappa=\frac{k_{\perp}^{2}}{\left|k^{2}\right|} \text { and } \tilde{\kappa}=1-\kappa .
$$

We note that the last integral was much more easily obtained by performing the $\kappa$ integration before the ones over the

\footnotetext{
${ }^{5}$ Here one obviously has to discard the overall factor $i$.
}

Feynman parameters. Therefore we only present the final, $\kappa$-integrated, result in this case. As can be seen, the integral was accompanied by two different powers of $\kappa$.

\section{APPENDIX B: THREE-PARTICLE PHASE SPACE}

As we discussed in Sec. I, the phase space for two gluons (plus one "observed" parton) will split up into four pieces for the ML prescription:

$$
\begin{aligned}
P S_{1}= & x \int d^{d} l_{1} d^{d} l_{2} \delta\left(1-x-\frac{n l_{1}+n l_{2}}{p n}\right) \delta\left(l_{1}^{2}\right) \delta\left(l_{2}^{2}\right), \\
P S_{2}= & x \int d^{d} l_{1} d^{d} l_{2} \delta\left(1-x-\frac{n l_{1}+n l_{2}}{p n}\right) \\
& \times \delta\left(l_{1}^{2}+l_{1, \perp}^{2}\right) \delta\left(l_{2}^{2}\right), \\
P S_{3}= & x \int d^{d} l_{1} d^{d} l_{2} \delta\left(1-x-\frac{n l_{1}+n l_{2}}{p n}\right) \\
& \times \delta\left(l_{1}^{2}\right) \delta\left(l_{2}^{2}+l_{2, \perp}^{2}\right), \\
P S_{4}= & x \int d^{d} l_{1} d^{d} l_{2} \delta\left(1-x-\frac{n l_{1}+n l_{2}}{p n}\right) \\
& \times \delta\left(l_{1}^{2}+l_{1, \perp}^{2}\right) \delta\left(l_{2}^{2}+l_{2, \perp}^{2}\right),
\end{aligned}
$$

where $l_{1}, l_{2}$ are the gluon momenta. The $\delta$ functions in Eqs. (B1) determine whether one (or both) of the gluons acts as an axial ghost.

As we know from the discontinuity in Eq. (4), the tensorial structures of the nonghost part and the ghost part are different. However, we can rewrite Eq. (4) as

$$
\begin{aligned}
\operatorname{disc}\left[\mathcal{D}^{\mu \nu}(l)\right]= & 2 \pi \Theta\left(l_{0}\right) \frac{2 n^{*} l}{l_{\perp}^{2}}\left[\delta\left(l^{2}\right)-\delta\left(l^{2}+l_{\perp}^{2}\right)\right] \\
& \times\left[-g^{\mu \nu}(n l)+n^{\mu} l^{\nu}+n^{\nu} l^{\mu}\right] .
\end{aligned}
$$

This is possible because of $2 n^{*} l / l_{\perp}^{2}=1 / n l$ for $l^{2}=0$ and $(n l)\left(n^{*} l\right)=0$ for $l^{2}+l_{\perp}^{2}=0$. In this way, it is always possible to calculate just one combined matrix element, using the tensorial structure in square brackets, and integrate it over a phase space subject to simply the difference $\delta\left(l^{2}\right)$ $-\delta\left(l^{2}+l_{\perp}^{2}\right)$. For our two-gluon case, this means that we have to consider only the combination

$$
P S_{1}-P S_{2}-P S_{3}+P S_{4} \text {. }
$$

We now introduce the Sudakov parametrizations

$$
\begin{aligned}
& l_{1}^{\mu}=(1-z) p^{\mu}+\frac{l_{1} p}{p n} n^{\mu}+l_{1, \perp}^{\mu}, \\
& l_{2}^{\mu}=z(1-y) p^{\mu}+\frac{l_{2} p}{p n} n^{\mu}+l_{2, \perp}^{\mu},
\end{aligned}
$$

where $\left(l_{i, \perp}^{\mu}\right)^{2}=-l_{i, \perp}^{2}$. The first $\delta$ functions in Eqs. (B1) imply $y=x / z$. If one wants to integrate over an arbitrary function $f$ of scalar products of the momenta, one writes the four parts of phase space in the following way: 
TABLE IV. Two- and three-point integrals with a light-cone gauge denominator for the ML prescription, calculated up to $\mathcal{O}(\epsilon)$. We have dropped the ubiquitous factor $i / 16 \pi^{2}\left(4 \pi /\left|k^{2}\right|\right)^{\epsilon} \Gamma(1-\epsilon) / \Gamma(1-2 \epsilon)$. $\tilde{x}$ and $\tilde{\kappa}$ have been defined in Eqs. (A9) and (A10), respectively.

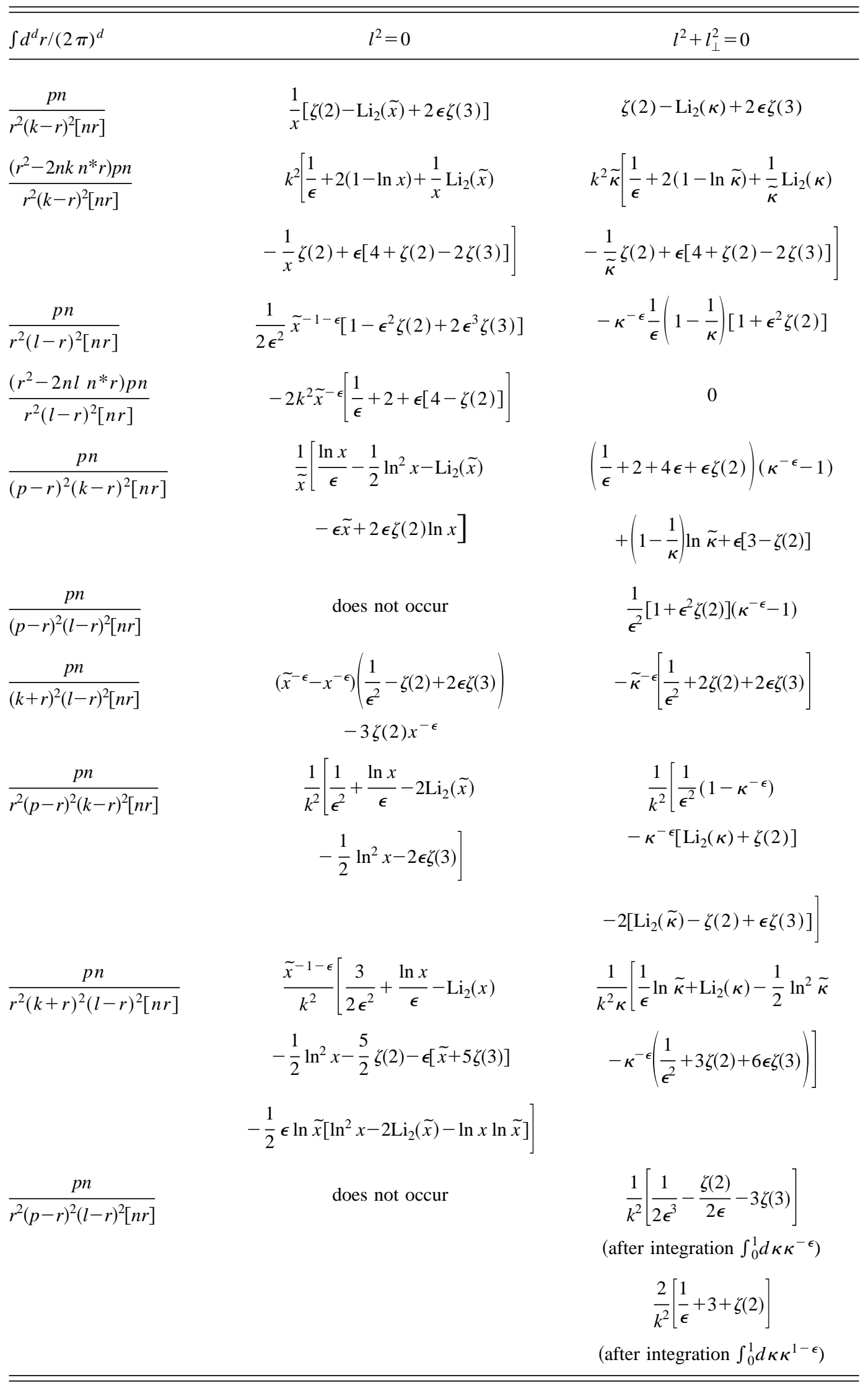




$$
\begin{aligned}
& x \int d^{d} l_{1} d^{d} l_{2} \delta\left(1-x-\frac{n l_{1}+n l_{2}}{p n}\right) f\left(\frac{l_{1, \perp}^{2}}{\left|k^{2}\right|}, \frac{-\left(p-l_{1}\right)^{2}}{\left|k^{2}\right|}, \frac{l_{2, \perp}^{2}}{\left|k^{2}\right|}, \frac{l_{2} p}{\left|k^{2}\right|}, x, z\right) \\
& \quad \times\left\{\delta\left(l_{1}^{2}\right) \delta\left(l_{2}^{2}\right)-\delta\left(l_{1}^{2}+l_{1, \perp}^{2}\right) \delta\left(l_{2}^{2}\right)-\delta\left(l_{1}^{2}\right) \delta\left(l_{2}^{2}+l_{2, \perp}^{2}\right)+\delta\left(l_{1}^{2}+l_{1, \perp}^{2}\right) \delta\left(l_{2}^{2}+l_{2, \perp}^{2}\right)\right\} \\
&= \frac{1}{4} \frac{\pi^{1-\epsilon}}{\Gamma(1-\epsilon)} \frac{\pi^{1 / 2-\epsilon}}{\Gamma\left(\frac{1}{2}-\epsilon\right)} \int_{0}^{Q^{2}} d\left|k^{2}\right|\left|k^{2}\right|^{1-2 \epsilon} x^{\epsilon} \int_{0}^{1} d v v^{-\epsilon} \int_{0}^{1} d w(w \tilde{w})^{-\epsilon} \int_{0}^{\pi} d \theta \sin ^{-2 \epsilon} \theta \\
& \times\left[\tilde { x } ^ { 1 - 2 \epsilon } \tilde { v } ^ { - \epsilon } f \left(\frac{\tilde{x}}{x} v w(1-v \tilde{x}), \frac{l_{1, \perp}^{2}}{\left|k^{2}\right| v \tilde{x}}, \frac{\tilde{x} \tilde{v}}{1-v \tilde{x}}\left(r_{1}^{2}+r_{2}^{2}-2 r_{1} r_{2} \cos \theta\right), \frac{l_{2, \perp}^{2}}{\left.2\left|k^{2}\right| \tilde{x} \tilde{v}, x, 1-v \tilde{x}\right)}\right.\right. \\
&-\frac{\tilde{x}^{-\epsilon}}{\tilde{v}} f\left(\frac{v w}{x}, \frac{w}{x}, \tilde{x}\left(r_{3}^{2}+r_{4}^{2}-2 r_{3} r_{4} \cos \theta\right), \frac{l_{2, \perp}^{2}}{2\left|k^{2}\right| \tilde{x}}, x, 1\right)-\frac{\tilde{x}^{-\epsilon}}{\tilde{v}} f\left(\frac{w \tilde{x}}{x}, \frac{w}{x}, r_{5}^{2}+r_{6}^{2}-2 r_{5} r_{6} \cos \theta, \frac{\tilde{w} \tilde{v}}{2 x}, x, x\right) \\
&\left.+\frac{\delta(1-x)}{\tilde{v}} \int_{0}^{1} d \rho \rho^{-2+\epsilon} \tilde{\rho}^{-\epsilon} f\left(\frac{w \tilde{\rho}}{\rho}, \frac{w}{\rho}, r_{7}^{2}+r_{8}^{2}-2 r_{7} r_{8} \cos \theta, \frac{\tilde{w} \tilde{v}}{2}, 1,1\right)\right],
\end{aligned}
$$

where

$$
\begin{aligned}
& \tilde{x}=1-x, \quad \tilde{v}=1-v, \quad \tilde{w}=1-w, \quad \tilde{\rho}=1-\rho, \\
& r_{1}=\sqrt{\tilde{w}}, \quad r_{2}=\sqrt{\frac{v w \tilde{v} \tilde{x}^{2}}{x}} \\
& r_{3}=\sqrt{\tilde{w}}, \quad r_{4}=\sqrt{\frac{v w \tilde{x}}{x}} \\
& r_{5}=\sqrt{\frac{w \tilde{x}}{x}}, \quad r_{6}=\sqrt{v \tilde{w}} \\
& r_{7}=\sqrt{\frac{w \tilde{\rho}}{\rho}}, \quad r_{8}=\sqrt{v \tilde{w}} .
\end{aligned}
$$

The meaning of the arguments of the function $f$ in the various parts of the phase space can be seen from the first line of Eq. (B5). We note that the last part of the phase space in Eq. (B5) is proportional to $\delta(1-x)$; it corresponds to the contribution where both gluons are axial ghosts.

We do not list the results for the numerous different phase space integrals one encounters. We just mention that one frequently needs the integral

$$
\begin{aligned}
\int_{0}^{\pi} d \theta \frac{\sin ^{-2 \epsilon} \theta}{r_{a}^{2}+r_{b}^{2}-2 r_{a} r_{b} \cos \theta}= & B\left(\frac{1}{2}-\epsilon, \frac{1}{2}\right)\left[\frac{1}{r_{a}^{2}}{ }_{2} F_{1}\left(1,1+\epsilon, 1-\epsilon ; \frac{r_{b}^{2}}{r_{a}^{2}}\right) \Theta\left(r_{a}^{2}-r_{b}^{2}\right)\right. \\
& \left.+\frac{1}{r_{b}^{2}}{ }_{2} F_{1}\left(1,1+\epsilon, 1-\epsilon ; \frac{r_{a}^{2}}{r_{b}^{2}}\right) \Theta\left(r_{b}^{2}-r_{a}^{2}\right)\right],
\end{aligned}
$$

where ${ }_{2} F_{1}(a, b, c ; z)$ denotes the hypergeometric function [29]. To expand in $\epsilon$, the relations

$$
\begin{aligned}
& { }_{2} F_{1}(1,1+\epsilon, 1-\epsilon ; z)=(1-z)^{-1-2 \epsilon} F_{2} F_{1}(-\epsilon,-2 \epsilon, 1-\epsilon ; z), \\
& { }_{2} F_{1}(a \epsilon, b \epsilon, 1-c \epsilon ; z)=1+a b \epsilon^{2}\left[\operatorname{Li}_{2}(z)+\epsilon c \operatorname{Li}_{3}(z)+\epsilon(a+b+c) \mathrm{S}_{1,2}(z)\right]+\mathcal{O}\left(\epsilon^{2}\right)
\end{aligned}
$$

are useful, where [28]

$$
\mathrm{Li}_{3}(z) \equiv \int_{0}^{1} \frac{\ln y \ln (1-z y)}{y} d y, \quad \mathrm{~S}_{1,2}(z) \equiv \frac{1}{2} \int_{0}^{1} \frac{\ln ^{2}(1-z y)}{y} d y
$$




\section{APPENDIX C: IMAGINARY PARTS OF LOOP INTEGRALS}

To see how to extract the imaginary part of a loop integral, let us go back to our example in Eq. (A4) for the case $q=l$ there. The integration over the Feynman parameter $s$ in Eq. (A4) is trivial and can be done immediately. Rather than performing straightaway the integration over $t$ to get the general result of the integral for arbitrary $l^{2}$, it is more convenient to include the $\tau$ integration of Eq. (24) in the calculation and carry it out first:

$$
\begin{aligned}
\int d \tau \tau^{-\epsilon} I(n, l)= & \frac{-i \Gamma(1+\epsilon)}{16 \pi^{2} \epsilon} \frac{1}{[n l]}\left(\frac{4 \pi}{\left|k^{2}\right|}\right)^{\epsilon}(1-x)^{-\epsilon} x^{\epsilon} \\
& \times \int_{0}^{1} d t t^{-1-\epsilon} \int_{\tau_{\min }}^{\tau_{\max }} d \tau \tau^{-\epsilon} \\
& \times\left\{[\tau(1-t x)-1]^{-\epsilon}\right. \\
& \left.-(1-t)^{-\epsilon}(\tau-1)^{-\epsilon}\right\}
\end{aligned}
$$

where we have used the definitions in Eqs. (25). To result in an imaginary part, ${ }^{6}$ the limits for the $\tau$ integration have to be chosen in such a way that those terms in Eq. (C1) that are raised to the power $-\epsilon$ become negative, i.e., $0<\tau<1 /(1$ $-t x)$ for the first term in square brackets in Eq. (C1) and $0<\tau<1$ for the second. The $\tau$ integrations become trivial then and lead to simple beta functions. Afterwards, the $t$ integration can be performed; the result is given in Table V where we also list other integrals that we encountered. As can be seen from Table V, we also needed some integrals with an extra factor $\tau$ or $(1-\tau)$ in the numerator. We do not consider the covariant integrals in Table $\mathrm{V}$ since in their case the extraction of the imaginary part is rather straightforward.

As we discussed in Sec. II B [see Eq. (27)], we have terms $\sim 1 / l^{2} \sim 1 /(1-\tau)$ in the calculation, resulting from the propagator of the gluon running into the loop, and to be treated according to the principal-value prescription. Therefore, we will also need integrals such as Eq. (C1) with an extra factor $1 /(1-\tau)$ in the integrand. Such integrals are in general much more difficult to calculate. A typical integral needed is

$$
\mathrm{PV}\left[\int_{0}^{1 /(1-t x)} \frac{d \tau}{1-\tau}\right]=\ln \left(\frac{1-t x}{t x}\right)
$$

The integrals with an extra denominator $1-\tau$ are also collected in Table V.

We finally emphasize that Table $\mathrm{V}$ does not contain all terms $\sim \epsilon$, but is in general correct only to $\mathcal{O}(1)$. The only $\mathcal{O}(\epsilon)$ terms that are fully accounted for in Table $\mathrm{V}$ are those $\sim \zeta(2)$. We have not consistently determined the other contributions $\sim \epsilon$, because this is a very hard task. Therefore, since terms like $(1-x)^{-1-\epsilon}$ will lead to further pole factors

${ }^{6} \mathrm{We}$ obviously do not take into account the overall factor $i$ here. $\sim \delta(1-x) / \epsilon$ via Eq. (12), we will not be able to calculate the finite amount of $\delta(1-x)$ in the final result for the $C_{F} N_{C}$ part of the two-loop splitting function, except for the contributions $\sim \zeta(2) \delta(1-x)$. However, the expressions in Table $\mathrm{V}$ are sufficient for checking graph by graph the cancellation of all pole terms proportional to $\delta(1-x)$, i.e., for proving the finiteness of the 2 PI kernels at $x=1$ in the ML prescription.

\section{APPENDIX D: MELLIN MOMENTS}

The Mellin moments of a function $f(x)$ are defined by

$$
f^{n} \equiv \int_{0}^{1} d x x^{n-1} f(x)
$$

As a result, the moments of a convolution $f \otimes g$ [see Eq. (32)] become the product of the moments of $f$ and $g$ :

$$
(f \otimes g)^{n}=f^{n} g^{n} .
$$

The moments of Eq. (31) are easily obtained using the formulas in the appendix of [30]. To invert the moments of the product $f^{n} g^{n}$ back to $x$-space, one needs some further moment expressions. Everything can be derived from the relations in [30], and from

$$
\begin{aligned}
\int_{0}^{1} d x x^{n-1} \ln ^{2}(1-x)= & \frac{1}{n}\left[S_{1}^{2}(n)+S_{2}(n)\right], \\
\int_{0}^{1} d x x^{n-1}\left[\frac{\ln ^{2}(1-x)}{1-x}\right]_{+}= & \frac{1}{n}\left[S_{1}^{2}(n)+S_{2}(n)\right]-\frac{1}{3} S_{1}^{3}(n) \\
& -S_{1}(n) S_{2}(n)-\frac{2}{3} S_{3}(n), \\
\int_{0}^{1} d x x^{n-1} \operatorname{Li}_{2}(x)= & \frac{1}{n} \zeta(2)-\frac{1}{n^{2}} S_{1}(n), \\
\int_{0}^{1} d x x^{n-1} \ln x \ln (1-x)= & \frac{1}{n}\left[S_{2}(n)-\zeta(2)\right]+\frac{1}{n^{2}} S_{1}(n), \\
\int_{0}^{1} d x x^{n-1} \frac{\ln x \ln (1-x)}{1-x}= & {\left[S_{2}(n)-\zeta(2)\right]\left(\frac{1}{n}-S_{1}(n)\right) } \\
& +\frac{1}{n^{2}} S_{1}(n)-S_{3}(n)+\zeta(3), \\
&
\end{aligned}
$$

where

$$
S_{k}(n) \equiv \sum_{j=1}^{n} \frac{1}{j^{k}}
$$


TABLE V. Imaginary parts 'IP्' ' of loop integrals for the ML prescription, after integration over the variable $\tau$ of the LO phase space (24). As before we have defined $\tilde{x}=1-x$. The integrals are in general only correct to $\mathcal{O}(1)$; see text. Note that terms $\sim(1-x)^{-\epsilon}$ must not be expanded in $\epsilon$, as further pole terms can arise via Eq. (12). We have dropped the ubiquitous factor $1 / 16 \pi^{2}\left(4 \pi /\left|k^{2}\right|\right)^{\epsilon} \Gamma(1-\epsilon) / \Gamma(1-2 \epsilon)$.

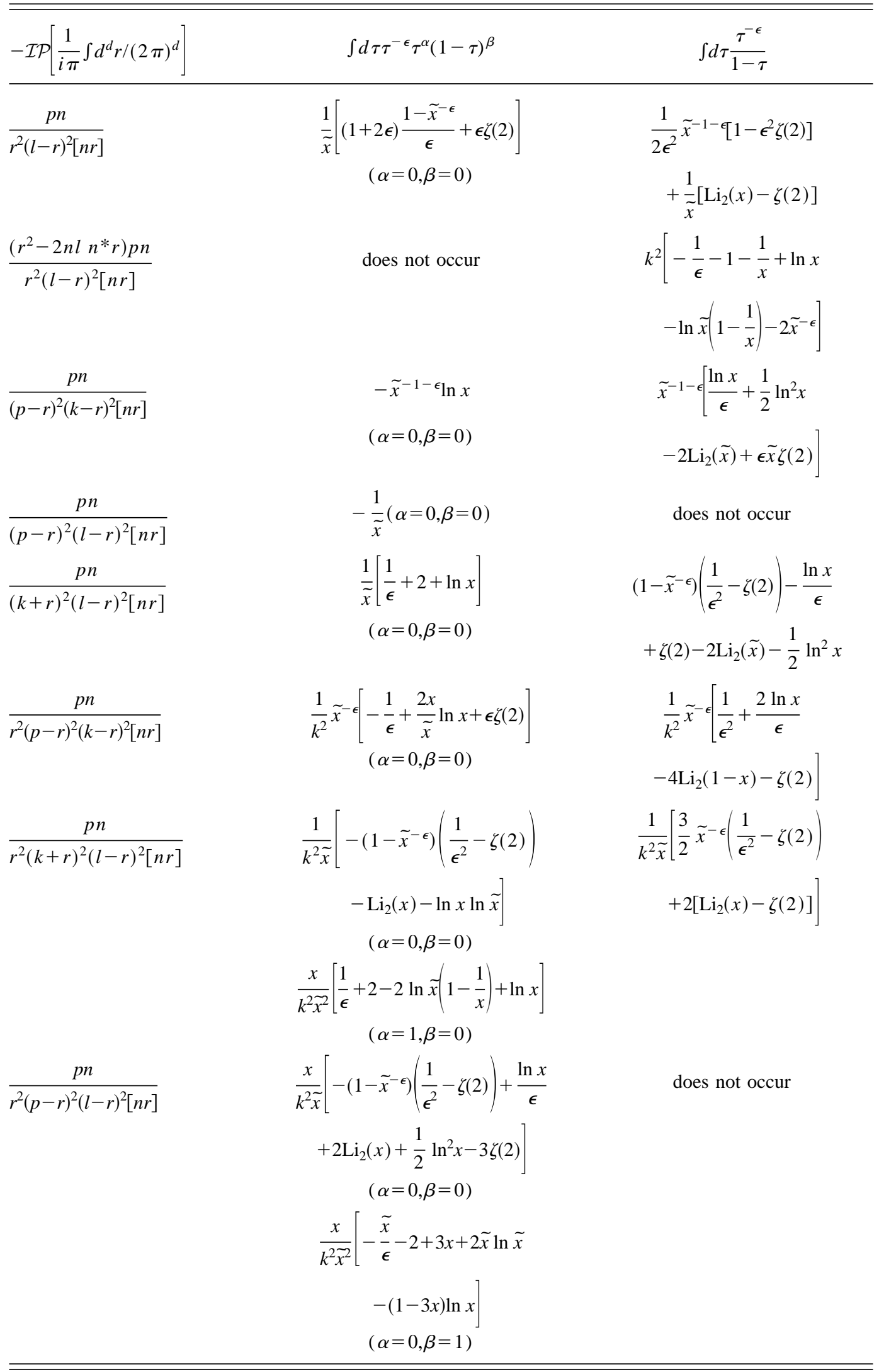




\section{APPENDIX E: TWO-LOOP INTEGRALS}

For the calculation of the $C_{F} T_{f}$ part of the two-loop quark self-energy we need some integrals with an extra noninteger power of $-r^{2}$ in the integrand, where $r$ is the loop momentum. Making use of the identities

$$
\begin{aligned}
\frac{1}{a^{\alpha} b} & =\alpha \int_{0}^{1} d x \frac{x^{\alpha-1}}{[a x+b(1-x)]^{\alpha+1}}, \\
\frac{1}{a^{\alpha} b c} & =\alpha(\alpha+1) \int_{0}^{1} d x \int_{0}^{1-x} d y \frac{x^{\alpha-1}}{[a x+b y+c(1-x-y)]^{\alpha+2}},
\end{aligned}
$$

one obtains, rather easily,

$$
\begin{gathered}
\int \frac{d^{d} r}{(2 \pi)^{d}} \frac{\left(-r^{2}\right)^{-\epsilon}}{(p-r)^{2}}=\frac{i}{16 \pi^{2}}(4 \pi)^{\epsilon}\left(-p^{2}\right)^{1-2 \epsilon} \frac{\epsilon \Gamma(2 \epsilon)}{\Gamma(1+\epsilon)} \frac{\Gamma(1-\epsilon) \Gamma(1-2 \epsilon)}{\Gamma(3-3 \epsilon)}, \\
\int \frac{d^{d} r}{(2 \pi)^{d}} \frac{\left(-r^{2}\right)^{-\epsilon}}{r^{2}(p-r)^{2}}=\frac{i}{16 \pi^{2}}(4 \pi)^{\epsilon}\left(-p^{2}\right)^{-2 \epsilon} \frac{\Gamma(2 \epsilon)}{\Gamma(1+\epsilon)} \frac{\Gamma(1-\epsilon) \Gamma(1-2 \epsilon)}{\Gamma(2-3 \epsilon)}, \\
\int \frac{d^{d} r}{(2 \pi)^{d}} \frac{\left(-r^{2}\right)^{-\epsilon}}{r^{2}(p-r)^{2}[n r]}=\left(-p^{2}\right)^{-\epsilon}\left[1+\epsilon^{2} \zeta(2)\right] \int \frac{d^{d} r}{(2 \pi)^{d}} \frac{1}{r^{2}(p-r)^{2}[n r]},
\end{gathered}
$$

where the integral on the right-hand side of Eq. (E4) was determined in Appendix A and is actually finite. For the ML prescription we therefore do not need the integral on the left-hand side of Eq. (E4); however, we will see below that the integral is divergent for the PV prescription. Also note that the integral in Eq. (E2) vanishes if the factor $\left(-r^{2}\right)^{-\epsilon}$ is not present.

Finally, for the PV prescription one obtains, for the integral in Eq. (E4),

$$
\int \frac{d^{d} r}{(2 \pi)^{d}} \frac{\left(-r^{2}\right)^{-\epsilon}}{r^{2}(p-r)^{2}[n r]}=\frac{i}{16 \pi^{2}} \frac{\Gamma(1+2 \epsilon)}{\Gamma(1+\epsilon)}(4 \pi)^{\epsilon}\left(-p^{2}\right)^{-2 \epsilon} \frac{1}{2 \epsilon p n}\left[I_{0}+\epsilon \zeta(2)-2 \epsilon I_{1}\right]+\mathcal{O}(\epsilon),
$$

while

$$
\int \frac{d^{d} r}{(2 \pi)^{d}} \frac{1}{r^{2}(p-r)^{2}[n r]}=\frac{i}{16 \pi^{2}} \Gamma(1+\epsilon)(4 \pi)^{\epsilon}\left(-p^{2}\right)^{-\epsilon} \frac{1}{\epsilon p n}\left[I_{0}+\epsilon \zeta(2)-\epsilon I_{1}\right]+\mathcal{O}(\epsilon) .
$$

Here

$$
I_{1} \equiv \int_{0}^{1} \frac{u \ln u}{u^{2}+\delta^{2}} d u
$$

[1] See, e.g., Yu. L. Dokshitzer, D. I. Dyakonov, and S. I. Troyan, Phys. Rep. 58, 270 (1980).

[2] A. Bassetto, G. Nardelli, and R. Soldati, Yang-Mills Theories in Algebraic Non-covariant Gauges (World Scientific, Singapore, 1991).

[3] A. Bassetto, M. Dalbosco, I. Lazzizzera, and R. Soldati, Phys. Rev. D 31, 2012 (1985).

[4] A. Bassetto, M. Dalbosco, and R. Soldati, Phys. Rev. D 36, 3138 (1987).

[5] S. Mandelstam, Nucl. Phys. B213, 149 (1983).

[6] G. Leibbrandt, Phys. Rev. D 29, 1699 (1984).
[7] A. Bassetto, in $Q C D$ and High Energy Hadronic Interaction, edited by J. Tran Than Van (Editions Frontières, Gif-surYvette, France, 1993); Nucl. Phys. B (Proc. Suppl.) 51C, 281 (1996).

[8] A. Bassetto, in "Proceedings of the VIII Warsaw Symposium on Elementary Particle Physics,' Kazimierz, Poland, 1985, edited by Z. Ajduk (Institute of Theoretical Physics, Warsaw University, Warsaw, 1985).

[9] G. Curci, W. Furmanski, and R. Petronzio, Nucl. Phys. B175, 27 (1980).

[10] W. Furmanski and R. Petronzio, Phys. Lett. 97B, 437 (1980). 
[11] R. K. Ellis, H. Georgi, M. Machacek, H. D. Politzer, and G. G. Ross, Phys. Lett. 78B, 281 (1978); Nucl. Phys. B152, 285 (1979).

[12] R. K. Ellis and W. Vogelsang, Report No. CERN-TH/96-50, RAL-TR-96-012, hep-ph/9602356.

[13] G. Altarelli and G. Parisi, Nucl. Phys. B126, 298 (1977).

[14] E. G. Floratos, D. A. Ross, and C. T. Sachrajda, Nucl. Phys. B152, 493 (1979); see also A. Gonzales-Arroyo and C. Lopez, ibid. B166, 429 (1980).

[15] R. Hamberg and W. L. van Neerven, Nucl. Phys. B379, 143 (1992); see also Y. Matiounine, J. Smith, and W. L. van Neerven, Phys. Rev. D 57, 6701 (1998).

[16] J. C. Collins and R. J. Scalise, Phys. Rev. D 50, 4117 (1994).

[17] G. Heinrich and Z. Kunszt, Nucl. Phys. B519, 405 (1998).

[18] G. Leibbrandt and S. L. Nyeo, Phys. Lett. 140B, 417 (1984).

[19] M. A. Nowak, M. Praszalowicz, and W. Slominski, Ann. Phys. (N.Y.) 166, 443 (1986).

[20] M. Dalbosco, Phys. Lett. B 180, 121 (1986).

[21] G. Leibbrandt, Rev. Mod. Phys. 59, 1067 (1987).
[22] E. G. Floratos, D. A. Ross, and C. T. Sachrajda, Nucl. Phys. B129, 66 (1977); B139, 545(E) (1978); see also A. GonzalesArroyo, C. Lopez, and F. J. Yndurain, ibid. B153, 161 (1979).

[23] R. T. Herrod and S. Wada, Phys. Lett. 96B, 195 (1980).

[24] G. Leibbrandt and J. Williams, Nucl. Phys. B440, 573 (1995).

[25] J. Williams, Ph.D. thesis, University of Guelph, 1998. We are grateful to J. Williams for communicating to us his results concerning the second and third diagram in the last row of Fig. 2.

[26] M. Dalbosco, Phys. Lett. 163B, 181 (1985).

[27] G. Leibbrandt, Noncovariant Gauges: Quantization of YangMills and Chern-Simons Theory in Axial Type Gauges (World Scientific, Singapore, 1994).

[28] A. Devoto and D. W. Duke, Riv. Nuovo Cimento 7, 1 (1984).

[29] I. S. Gradshteyn and I. M. Ryzhik, Table of Integrals, Series, and Products (Academic, London, 1979).

[30] G. Altarelli, R. K. Ellis, and G. Martinelli, Nucl. Phys. B157, 461 (1979). 